\title{
Excitation of airwaves by bubble bursting in suspensions: regime transitions and implications for basaltic volcanic eruptions
}

\author{
Kana Hashimoto and Ikuro Sumita* (1)
}

\begin{abstract}
Basaltic magma becomes more viscous, solid-like (elastic), and non-Newtonian (shear-thinning, non-zero yield stress) as its crystal content increases. However, the rheological effects on bubble bursting and airwave excitation are poorly understood. Here we conduct laboratory experiments to investigate these effects by injecting a bubble of volume $V$ into a refractive index-matched suspension consisting of non-Brownian particles (volumetric fraction $\phi$ ) and a Newtonian liquid. We show that depending on $\phi$ and $V$, airwaves with diverse waveforms are excited, covering a frequency band of $f=\mathcal{O}\left(10-10^{4}\right) \mathrm{Hz}$. In a suspension of $\phi \leq 0.3$ or in a suspension of $\phi=0.4$ with a $V$ smaller than critical, the bubble bursts after it forms a hemispherical cap at the surface and excites a high-frequency (HF) wave ( $f \sim 1-2 \times 10^{4} \mathrm{~Hz}$ ) with an irregular waveform, which likely originates from film vibration. However, in a suspension of $\phi=0.4$ and with a $V$ larger than critical, the bubble bursts as soon as it protrudes above the surface, and its aperture opens slowly, exciting Helmholtz resonance with $f=\mathcal{O}\left(10^{3}\right) \mathrm{Hz}$. Superimposed on the waveform are an HF wave component excited upon bursting and a low-frequency $(f=\mathcal{O}(10) \mathrm{Hz})$ air flow vented from the deflating bubble, which becomes dominant at a large $V$. We interpret this transition as a result of the bubble film of a solid-like $\phi=0.4$ suspension, being stretched faster than the critical strain rate such that it bursts by brittle failure. When the Helmholtz resonance is excited by a bursting bubble in a suspension whose surface level is further below the conduit rim, an air column (length $L$ ) resonance is triggered. For $L$ larger than critical, the air column resonance continues longer than the Helmholtz resonance because the decay rate of the former becomes less than that of the latter. The experiments suggest that a bubble bursting at basaltic volcanoes commonly excites HF wave by film vibration. The Helmholtz resonance is likely to be excited under a limited condition, but if detected, it may be used to track the change of magma rheology or bubble $V$, where the $V$ can be estimated from its frequency and decay rate.
\end{abstract}

Keywords: Magmatic suspension, Viscous-brittle transition, Bubble bursting, Broad frequency band, High-frequency wave, Helmholtz resonance, Air column resonance, Air flow

\section{Introduction}

In Strombolian and Hawaiian eruptions, bubbles burst upon surfacing and excite airwaves whose main energy is contained in the infrasonic band (Johnson and Ripepe 2011; Fee and Matoza 2013). Magma commonly

\footnotetext{
*Correspondence: isumita@staff.kanazawa-u.ac.jp

Earth and Environmental Science Course, Division of Natural System,

Graduate School of Natural Science and Technology, Kanazawa University, Kanazawa 920-1192, Japan
}

contains crystals (e.g., Gurioli et al. (2014)), which not only increase the viscosity but also give rise to viscoelasticity and non-Newtonian rheology (shear-thinning, nonzero yield stress) (Mader et al. 2013; Namiki and Tanaka 2017). Such complex rheology is considered to affect the style of bubble bursting and airwave excitation, but their details are unknown.

In order to better understand how such complex rheology controls bubble bursting and airwave excitation, laboratory experiments have been conducted by 
injecting an air bubble or continuous flow of air into a non-Newtonian gel and analyzing the recorded airwave (Divoux et al. 2008; Vidal et al. 2009; Lyons et al. 2013). These studies have shown that the characteristic frequencies of the airwaves depend on the bubble volume and revealed temporal switching of the style of outgassing and airwave excitation. The magma surface at which the bubble bursts is usually below the conduit rim. Previous experiments have also modeled such situation and have shown that air column resonance can be excited in the conduit above the bubble (James et al. 2004; Kobayashi et al. 2010). Experiments have also shown that resonance can be excited in the cavity below the ruptured film (Vidal et al. 2006, 2010). These results indicate that airwaves excited by different mechanisms may be superimposed and that we need to distinguish them. Although previous experiments using gels have been conducted by varying their concentrations which change their rheology, it remained unclear how this corresponded to the crystal content in the magma. A more direct way to model the effects of crystals is to use suspensions, which however are usually opaque. In this study we match the refractive indices of particles and the liquid (Wiederseiner et al. 2011), which allows us to visualize the subsurface bubble.

This paper is organized as follows. First we describe the rheology of the suspensions we use for our experiments. Next we show how the styles of bubble bursting and airwave excitation transition as a function of the particle volumetric fraction $\phi$ of the suspension and bubble volume $V$. We analyze the high-speed images and waveforms of the airwaves to infer the excitation mechanisms. Then we lower the suspension level below the tank rim to study how the air column resonance is simultaneously excited, and obtain the critical air column length above which it determines the duration of the airwave. Finally, we provide implications for basaltic volcanic eruptions.

\section{Physical properties of suspensions}

For the particles, we use spherical silicone powder (ShinEtsu Chemical, KMP-590) with a diameter of $d_{\mathrm{p}} \simeq 2 \mu \mathrm{m}$ and a density of $\rho_{\mathrm{p}}=1300 \mathrm{~kg} / \mathrm{m}^{3}$. We prepare a silicone oil mixture that has a refractive index close to that of the silicone powder, such that the suspension becomes clearest at $23^{\circ} \mathrm{C}$. The oil has a density of $\rho_{\mathrm{l}}=984.2$ $\mathrm{kg} / \mathrm{m}^{3}$, a Newtonian viscosity of $\eta_{1}=0.1$ Pas, and a surface tension coefficient of $\sigma \simeq 0.021 \mathrm{~N} / \mathrm{m}$ (Shin-Etsu Chemical). The particle - liquid density difference is $\Delta \rho_{\mathrm{pl}}=\rho_{\mathrm{p}}-\rho_{l}=315.8 \mathrm{~kg} / \mathrm{m}^{3}$. Further details on the particles, oils, the effects of gravity, thermal agitation, viscous and inertial stresses on the particle-scale motions under the strain rate of bubble ascent and rheometry, are given in the Additional file 7: Material.

In comparison, the $\rho_{\mathrm{p}}$ of the crystals consisting the magmatic suspension (e.g., olivine, pyroxene, plagioclase (Gurioli et al. 2014)) is in the range of $\rho_{\mathrm{p}}=2630-3700$ $\mathrm{kg} / \mathrm{m}^{3}$, and the density of basaltic magma is $\rho=2600-2650 \mathrm{~kg} / \mathrm{m}^{3}$ (Philpotts and Ague 2009). Thus the $\Delta \rho_{\mathrm{pl}}=\mathcal{O}\left(10-10^{3}\right) \mathrm{kg} / \mathrm{m}^{3}$ overlaps the $\Delta \rho_{\mathrm{pl}}$ of our suspension. A finite $\Delta \rho_{\mathrm{pl}}$ is a cause for the non-Brownian nature and the yield stress (Fall et al. 2009) of these suspensions.

We thoroughly mix the particles and the oil to form suspensions with volumetric packing fractions of $\phi=$ $0.10,0.30,0.40$, and 0.51 . We hereafter use $\phi$ rounded to 1 digit to denote a suspension with a specified $\phi$. The bulk densities are $\rho=1016.1,1082.5,1110.3$, and 1143.7 $\mathrm{kg} / \mathrm{m}^{3}$ for suspensions of $\phi=0.1,0.3,0.4$, and 0.5 , respectively. The $\sigma$ of suspensions of $\phi=0.3$ and 0.4 measured using a pendant drop technique are $\sim 20 \%$ smaller than that of particle-free oil.

\section{Rheology of suspensions}

We measured the rheology of suspensions using a rheometer (Anton-Paar, MCR 301) in cone-plate geometry (Additional file 7: Material). Figure 1a shows the flow

\footnotetext{
(See figure on next page.)

Fig. 1 Rheology of suspensions (packing fraction $\phi$ ). a Flow curves and their fits to Eq. (1). Fitting parameters are as follows; $\phi=0: \tau_{y}=0$ $(\mathrm{Pa}), n=0.998, K=0.10\left(\mathrm{~Pa} \mathrm{~s}^{n}\right), \phi=0.1: \tau_{y}=0.07(\mathrm{~Pa}), n=0.96, K=0.14\left(\mathrm{~Pa}^{n}\right), \phi=0.3: \tau_{y}=1.2(\mathrm{~Pa}), n=0.91, K=0.50\left(\mathrm{~Pa} s^{n}\right)$, $\phi=0.4: \tau_{\mathrm{y}}=4.7(\mathrm{~Pa}), n=0.87, K=2.0\left(\mathrm{~Pa} s^{n}\right)$, and $\phi=0.5: \tau_{\mathrm{y}}=27(\mathrm{~Pa}), n=0.75, K=19.2\left(\mathrm{~Pa} \mathrm{~s}^{n}\right)$. $\mathbf{b}$ The $\phi$ dependence of relative viscosity $\eta_{\mathrm{r}}=\eta / \eta_{1}$. A red curve indicates $\eta_{\mathrm{r}}$ calculated from Eq. (2) with $\phi_{\mathrm{c}}=0.60$ (indicated by a blue broken line). c Stress sweeps of storage ( $G^{\prime}$ : large markers) and loss ( $G^{\prime \prime}$ : small markers) moduli measured under small to large amplitude oscillatory shear at $f=1 \mathrm{~Hz}$. Arrows indicate the linear and non-linear regions of the $\phi=0.4$ suspension. $G^{\prime} \simeq 0$ of $\phi=0,0.1$ suspensions are not plotted. $x$ indicates the $G^{\prime}, G^{\prime \prime}$ crossover which defines the yield stress $\tau_{y}$ (corresponding to a strain $\gamma \sim 4 \times 10^{-3}$ ). $\mathbf{d}$ Frequency sweeps of $G^{\prime}$ (large markers) and $G^{\prime \prime}$ (small markers), measured under a small amplitude $\left(\gamma=10^{-4}\right)$ oscillatory shear. The thick (thin) broken line indicates the instrumental inertia effect ( $G_{\text {inertia }}$ ) of the spindle used to measure the $\phi=0$ ( $\phi \geq 0.1$ ) suspension (Additional file 7: Eq. (3)). e Creep curves of $\phi=0.4$ suspension sheared under the $\tau$ indicated in the legend. $\tau$ was applied during $0 \leq t \leq 5 \mathrm{~s}$ and released at $t=5 \mathrm{~s}$ (response time $<0.01 \mathrm{~s}$ ). The $\gamma$ is normalized by the respective maximum values $\gamma$ max at $t \simeq 5 \mathrm{~s}$, which increases from $\gamma_{\max }=1.4 \times 10^{-3}(\tau=0.2 \mathrm{~Pa})$ to $\gamma_{\max }=6.17(\tau=5 \mathrm{~Pa}) . \mathbf{f} \phi$ dependence of the yield stress $\tau_{\mathrm{y}}$ (with errors) obtained from the flow curves (a) and stress sweeps (c). The bubble buoyancy pressures $p$ (Eq. (5)) are plotted for comparison
} 

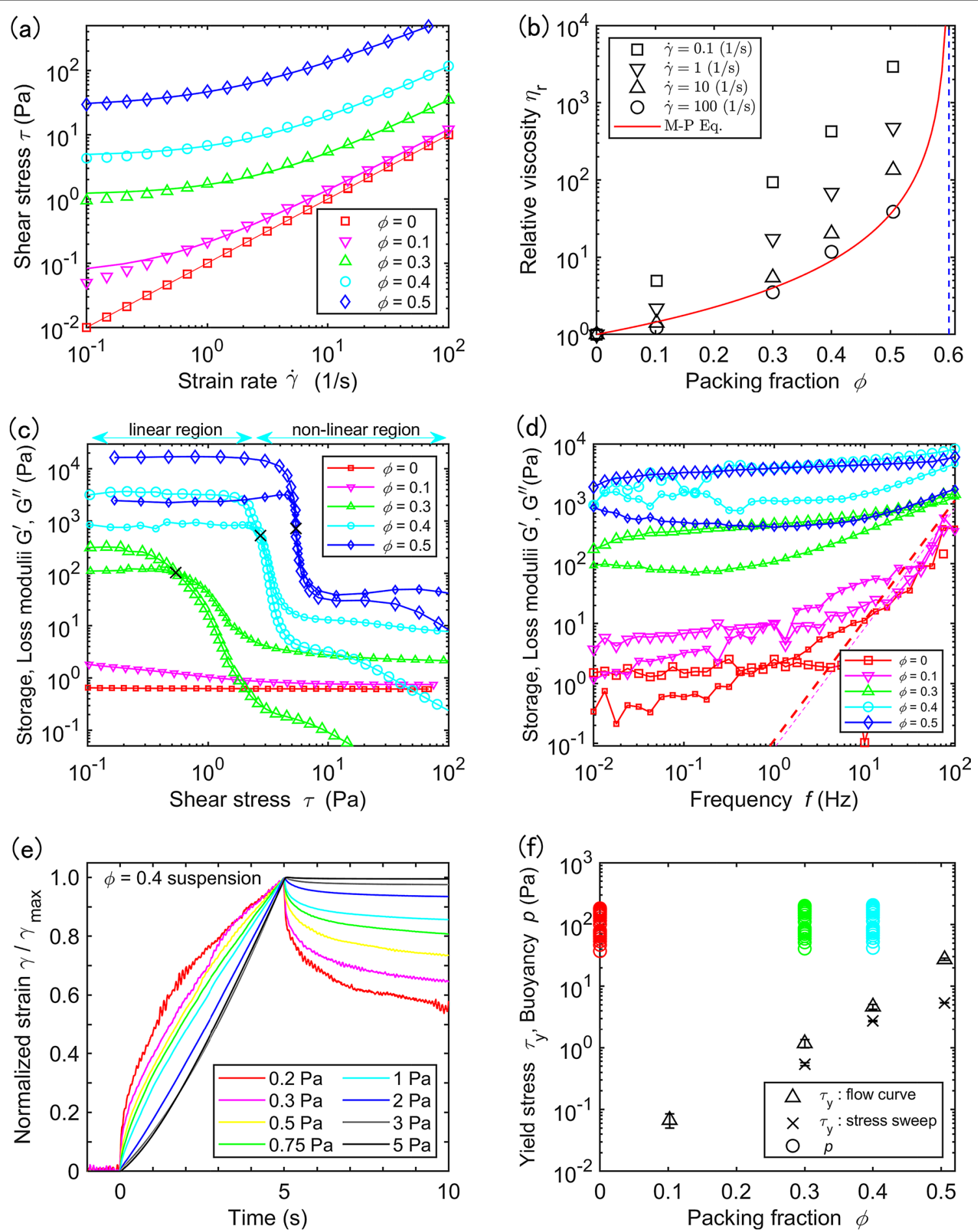

Fig. 1 (See legend on previous page.)

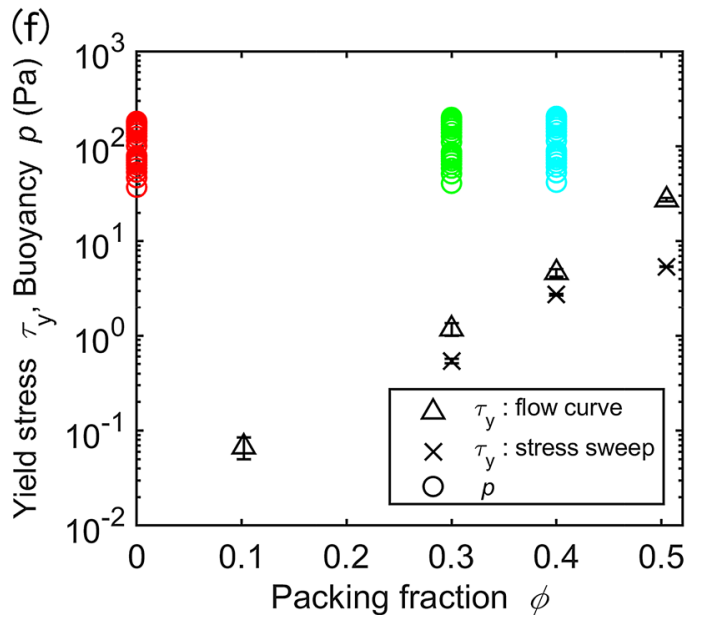

curves (stress $\tau$ vs. strain rate $\dot{\gamma}$ ). We fit the plots to a Herschel-Bulkley model (Mader et al. 2013)

$$
\tau=\tau_{\mathrm{y}}+K \dot{\gamma}^{n},
$$

where $\tau_{\mathrm{y}}$ is the yield stress, $K$ is the consistency, and $n$ is the flow index (see legend for fitted values). $n$ decreases from $n \simeq 1.00$ (Newtonian) for oil $(\phi=0)$ to $n=0.75$ 
(shear-thinning) for a suspension of $\phi=0.5$. These values of $n$ are similar to those of basaltic magma (Tran et al. (2015), Additional file 7: Material). Viscosity $\eta$ is calculated from $\eta=\tau / \dot{\gamma}$ (see Additional file 7: Fig. S3 for $\eta$ vs. $\dot{\gamma}$ plot). Figure $1 \mathrm{~b}$ shows relative viscosity $\eta_{\mathrm{r}}=\eta / \eta_{\mathrm{l}}$ $\left(\eta_{1}\right.$ : liquid viscosity) vs. $\phi$. We fit the measurements at $\dot{\gamma}=100 \mathrm{1} / \mathrm{s}$ using the Maron-Pierce correlation (Guazzelli and Pouliquen 2018),

$$
\eta_{\mathrm{r}}=\left(1-\frac{\phi}{\phi_{\mathrm{c}}}\right)^{-2}
$$

where $\phi_{\mathrm{c}}=0.60$ (maximum packing fraction) is the fitting parameter. The fit using the Einstein-Roscoe equation results in $\phi_{\mathrm{c}}=0.66$, a value larger than the random close packing of spheres $\phi_{\mathrm{c}} \simeq 0.64$.

Figure 1c shows the stress sweeps of storage and loss moduli $\left(G^{\prime}, G^{\prime \prime}\right)$ measured under a small to large amplitude oscillatory shear. The $\phi=0,0.1$ suspensions are liquid-like $\left(G^{\prime \prime} \gg G^{\prime}\right)$ and we do not plot $G^{\prime}$. The $\phi \geq 0.3$ suspensions are solid-like $\left(G^{\prime}>G^{\prime \prime}\right)$ at small $\tau$ and the $G^{\prime}, G^{\prime \prime}$ do not depend on $\tau$ (the linear region), but fluidize $\left(G^{\prime \prime}>G^{\prime}\right)$ at large $\tau$ (the non-linear region). We classify the $\phi \geq 0.3(\phi \leq 0.1)$ suspensions as solidlike (liquid-like) using the results at small $\tau$, though we emphasize that solid-like suspensions become liquidlike at large $\tau$ (Sumita and Manga 2008). For solid-like suspensions, we define the yield stress $\tau_{\mathrm{y}}$ as the $\tau$ at which the $G^{\prime}, G^{\prime \prime}$ crossover (Bonn et al. 2017).

Figure $1 \mathrm{~d}$ shows the frequency sweeps of $G^{\prime}, G^{\prime \prime}$ measured under a small amplitude $\left(\gamma=10^{-4}\right)$ oscillatory shear, in the linear region for the $\phi \geq 0.3$ suspensions. For $\phi=0,0.1$, since $G^{\prime \prime} \propto f$, the rheology is liquid-like (viscous) (Larson 1999) (see Additional file 7: Material 3 and 4.3 for other effects). On the other hand, for $\phi=0.3-0.5$, since $G^{\prime}>G^{\prime \prime}$ and $G^{\prime}$ becomes nearly frequency independent, indicating a solid-like (elastic) rheology.

The rheology shown in Fig. 1a-d were obtained under steady or oscillatory states. Now we show a transient response. Figure 1e shows the creep curves of a solid-like $\phi=0.4$ suspension when $\tau$ is applied and released. Since the strain $\gamma$ increases with $\tau$, we normalized the $\gamma$ by their respective maximum values $\gamma_{\max }$. At small $\tau$, an initial elastic response (a rescaled close-up is shown in Additional file 7: Fig. S4) is followed by a viscous flow, and an elastic recovery after the stress release. However at large $\tau$, the viscous strain becomes dominant. This result indicates that the rheology can be most simply approximated as a shear-thinning Maxwell fluid consisting of a viscous component $\eta(\dot{\gamma})$ and an infinite frequency elastic component $G_{\infty}$. As $\tau$ (hence $\dot{\gamma}$ ) increases, the $\eta(\dot{\gamma})$ decreases, and the relaxation time $t_{\mathrm{r}}=\eta(\dot{\gamma}) / G_{\infty}$, the time scale in

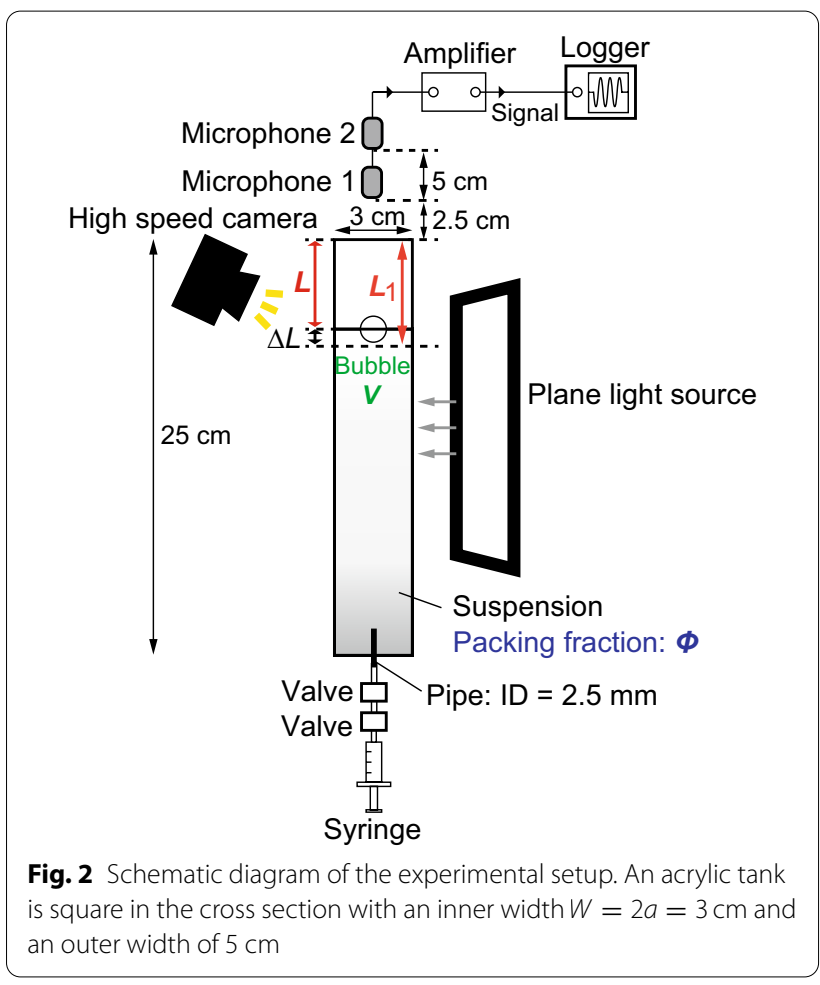

which the elastic and viscous strains become comparable, becomes shorter. This rheology is similar to that of a magmatic suspension which is also shear-thinning (e.g., Ishibashi (2009); Gurioli et al. (2014)) and Maxwellian (viscous creep at $t>t_{\mathrm{r}}$; e.g., Cordonnier et al. (2012)).

Figure 1f shows $\tau_{\mathrm{y}}$ vs. $\phi$ obtained from the flow curves (Fig. 1a) and stress sweeps (Fig. 1c). The $\tau_{\mathrm{y}}$ obtained from the 2 methods agree within a factor of $2-5$.

\section{Experimental method}

Figure 2 shows the experimental setup. We inject a bubble from the bottom of a square tank and record images of the bubble ascent and bursting near the surface using a high-speed camera (IDT, M3) at $3000 \mathrm{fps}$ (for runs using suspensions of $\phi=0,0.3$ ) and $2000 \mathrm{fps}$ (for runs using suspension of $\phi=0.4$ and $\eta=3.2,10.8$ Pas oil). The excited airwave is recorded using 2 vertically aligned microphones (Primo, MX5307 ; sensitivities 5.7, 5.4 mV/ $\mathrm{Pa})$ with a flat response in the frequency band of 10 $20000 \mathrm{~Hz}$ (calibrated from $0.5 \mathrm{~Hz}$ ). The voltage outputs of the microphones are amplified by $\times 74.645$ (Primo, MX5002) and recorded at $1 \mathrm{MHz}$ using a data logger (Graphtec, GL980) synchronized to high-speed imagery. We mainly analyze the large-amplitude data recorded by the lower microphone 1 closer to the bubble. Unless noted otherwise, waveforms shown are those recorded by Mic 1 . The signal recorded by the upper microphone 
2 is used to study the time lag and decay of the airwave. These microphones are located in the near or far field of the wavefield, depending on the frequency and excitation mechanism (Additional file 7: Material). We checked the response of our microphones to a pressure pulse generated by a hand fan. We confirmed that in addition to the sound wave, the microphone detects the pressure fluctuation arising from the air flow with a velocity $v_{\text {af }}=\mathcal{O}(1)$ $\mathrm{m} / \mathrm{s}$ and $f \sim 20 \mathrm{~Hz}$ and that the waveforms recorded by the 2 adjacent microphones correlate well. The room temperature is maintained close to $23^{\circ} \mathrm{C}$ so that the suspension remains as clear as possible.

In the first series of experiments, we use a nearly full tank and vary the $\phi$ of the suspensions $(\phi=0,0.3,0.4)$ and bubble volume $V\left(V=0.1-12 \mathrm{~cm}^{3}\right)$. We also conduct experiments using high viscosity $(\eta=3.2,10.8$ Pas $)$ silicone oils to compare with the results using suspensions. The level $L_{1}$ before the bubble injection is specified as follows. When the bubble is injected, the fluid level rises by $\Delta L$ (see Fig. 2), and the air column length $L$ between the tank rim and the fluid surface decreases as $L=L_{1}-\Delta L$. Accordingly we specified $L_{1}$ such that $L$ becomes $L \sim 0-1 \mathrm{~cm}$ after the bubble is injected. In the second series of experiments, we study $L$ dependence by injecting a $V=5 \mathrm{~cm}^{3}$ bubble into a suspension of $\phi=0.4$. We lower the level in 7 steps in the range of $L_{1}=$ $1-10 \mathrm{~cm}$. $L$ is similarly calculated by correcting for $\Delta L$.

\section{Bubble size, overpressure and buoyancy}

The bubble size is characterized by an equivalent bubble diameter $d_{\text {be }}$ (or radius $R_{\text {be }}$ ) defined as

$$
d_{\text {be }}=2 R_{\text {be }}=\left(\frac{6 V}{\pi}\right)^{1 / 3} .
$$

The $d_{\text {be }}$ values of the bubbles we inject are $d_{\text {be }}=5.8-28.4 \mathrm{~mm}$, which when normalized to the tank width $W$, become $d_{\text {be }} / W=0.19-0.95$. For $d_{\text {be }} / W>0.6$ (corresponding to a large bubble of $V>3$ $\mathrm{cm}^{3}$ ), the bubble becomes vertically elongated to form a slug (Clift et al. 1978). We note that $d_{\text {be }}$ is more than 3 orders of magnitude larger than the diameter of the particles $\left(d_{\mathrm{p}} \simeq 2 \mu \mathrm{m}\right)$ suspended in the oil. This indicates that the suspension can be considered a homogeneous medium for the rising bubble. The overpressure $\Delta P$ of these bubbles is estimated as $\Delta P \sim 2 \sigma / R_{\mathrm{be}}=1-8 \mathrm{~Pa}$.

A dimensionless form of $d_{\text {be }}$ is the Eötvös (or Bond) number

$$
E o=B o=\frac{\Delta \rho g d_{\mathrm{be}}^{2}}{\sigma},
$$

which compares buoyancy ( $\Delta \rho$ : suspension - air density difference) to capillary stress. Eo governs the

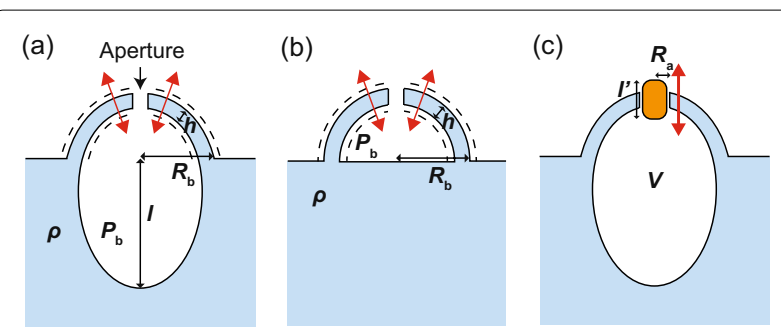

Fig. 3 Schematic diagrams showing several mechanisms of airwave excitation. a Film vibration of a bubble with a hemispherical cap and a hemispheroid below the surface. $\mathbf{b}$ Film vibration of a hemispherical cap bubble. $\mathbf{c}$ Helmholtz resonance at the neck (effective length $I^{\prime}$ ) of an aperture (radius $R_{a}$ )

shape of a static bubble protruding above the surface and the mechanism of bubble film drainage (Nguyen et al. 2013). For $E o \gg 1$, relevant to our experiments $\left(E o=\mathcal{O}\left(10^{1}-10^{2}\right)\right)$, a static bubble forms a hemispherical cap, and the film drainage is gravity driven (Additional file 7: Material). Similarly, the Eo of a $d=1$ $\mathrm{m}$ bubble in basaltic magma is Eo $\sim 7 \times 10^{4} \gg 1$, where we used $\rho=2650 \mathrm{~kg} / \mathrm{m}^{3}$ and $\sigma \sim 0.35 \mathrm{~N} / \mathrm{m}$ (Murase and McBirney 1973).

Bubble buoyancy pressure $p$ is evaluated from

$$
p=\frac{\Delta \rho g V}{\pi d_{\mathrm{be}}^{2} / 4}=\frac{2}{3} \Delta \rho g d_{\mathrm{be}}
$$

In Fig. 1f, we indicate the range of $p$ and compare with the yield stress $\tau_{\mathrm{y}}$. We find that $p$ is larger than $\tau_{\mathrm{y}}$ by at least a factor of $6-10$. This is consistent with the observation that the bubbles rise without being trapped.

\section{Mechanisms of airwave excitation and decay}

Here we summarize the mechanisms of airwave excitation and decay which are relevant to our experiments. First we consider film vibration. Figure 3a shows film vibration excited by a bursting bubble that partially protrudes above the surface. The following derivation is essentially the same as those of previous works (Vergniolle and Brandeis 1994; Kobayashi et al. 2010; Lyons et al. 2019), and is modified from the vibration of a fully immersed bubble (Leighton 1994). We consider a bubble consisting of a hemispherical cap (radius $R_{\mathrm{b}}$ ) above the surface and a hemispheroid (length $l$ ) below the surface. Then the total height of the bubble $R_{\mathrm{b}}+l$ becomes $R_{\mathrm{b}}+l=(3 V / 2 \pi)^{1 / 3}\left(1+l / R_{\mathrm{b}}\right)^{2 / 3}$. We assume that a hemispherical film with a thickness of $h$ and a density of $\rho$ vibrates while the fluid below the surface is rigid. From the balance between the kinetic energy $\pi R_{\mathrm{b}}^{2} \rho h(\omega \delta R)^{2}$ and potential energy $3 \pi \gamma_{\mathrm{h}} P_{\mathrm{b}}\left(R_{\mathrm{b}}^{2} /\left(R_{\mathrm{b}}+l\right)\right) \delta R^{2}$ ( $\delta R$ : amplitude of oscillation; $\omega$ : angular frequency; $\gamma_{\mathrm{h}}$ : ratio of specific 
heats; $P_{\mathrm{b}}$ : pressure inside the bubble), the film vibration frequency $f_{\mathrm{V}}$ becomes

$$
\begin{aligned}
& f_{\mathrm{V}}=\frac{1}{2 \pi} \sqrt{\frac{3 \gamma_{\mathrm{h}} P_{\mathrm{b}}}{\rho h\left(R_{\mathrm{b}}+l\right)}} \\
& =\frac{3^{1 / 3}}{(2 \pi)^{5 / 6}}\left(\frac{\gamma_{\mathrm{h}} P_{\mathrm{b}}}{\rho h}\right)^{1 / 2}\left(1+\frac{l}{R_{\mathrm{b}}}\right)^{-1 / 3} V^{-1 / 6} .
\end{aligned}
$$

Here we neglected an aperture and assumed that the whole film above the surface with a uniform thickness of $h$ vibrates. When $h$ is not uniform or if a localized sector of the film vibrates, then $h$ should be regarded as an effective value. $h$ can be estimated from the aperture growth velocity which we show later. For our experiments, $\gamma_{\mathrm{h}}=1.4$ (air) and $P_{\mathrm{b}}=1.01325 \times 10^{5} \mathrm{~Pa}$.

Figure $3 \mathrm{~b}$ shows a situation where a hemispherical cap bubble excites film vibration. $f_{\mathrm{V}}$ can be derived from Eqs. (6),(7) using $l=0$ as

$$
\begin{aligned}
& f_{\mathrm{V}}=\frac{1}{2 \pi} \sqrt{\frac{3 \gamma_{\mathrm{h}} P_{\mathrm{b}}}{\rho h R_{\mathrm{b}}}} \\
& =\frac{3^{1 / 3}}{(2 \pi)^{5 / 6}}\left(\frac{\gamma_{\mathrm{h}} P_{\mathrm{b}}}{\rho h}\right)^{1 / 2} V^{-1 / 6} .
\end{aligned}
$$

Second, we consider an airwave excited by instant removal of the bubble film (Vergniolle and Brandeis 1994). For an ideal spherical balloon, an $\mathrm{N}$-shaped wave is excited (Deihl and Carlson 1968), whose frequency $f_{\mathrm{N}}$ is expressed as

$$
f_{\mathrm{N}}=\frac{c}{2 R_{\mathrm{b}}} \propto V^{-1 / 3},
$$

where $c$ is the sound velocity. For our experiments, we use $c=345.3 \mathrm{~m} / \mathrm{s}$ at $23^{\circ} \mathrm{C}$. For a hemispherical cap, $R_{\mathrm{b}}$ becomes $R_{\mathrm{b}}=(3 V / 2 \pi)^{1 / 3}$.

Third, we consider Helmholtz resonance where the air within the neck of the aperture of a bubble vibrates (Fig. 3c), and has been used to model volcanic infrasound (Vergniolle and Caplan-Aucherbach 2004; Montalto et al. 2010). The Helmholtz resonance frequency $f_{\mathrm{H}}$ is expressed as (Kinsler et al. 2000),

$$
f_{\mathrm{H}}=\frac{c R_{\mathrm{a}}}{2} \sqrt{\frac{1}{\pi l^{\prime} V}}
$$

where $R_{\mathrm{a}}$ is the aperture radius and $l^{\prime}$ is the effective neck length. The $R_{\mathrm{a}}$ dependence in Eq. (11) arises from the restoring pressure of a compressible gas scaling as $\propto R_{\mathrm{a}}^{2} \cdot l^{\prime}$ is determined by the end correction of the aperture and scales as $l^{\prime} \propto R_{\mathrm{a}}$. From the estimate
$8 R_{\mathrm{a}} / 3 \pi \leq l^{\prime} \leq 16 R_{\mathrm{a}} / 3 \pi$ (Spiel 1992), we use $l^{\prime}=4 R_{\mathrm{a}} / \pi$ as a representative value. It follows that $f_{\mathrm{H}} \propto R_{\mathrm{a}}^{1 / 2}$ and $f_{\mathrm{H}}$ increases as $R_{\mathrm{a}}$ grows. A frequency gliding resulting from the aperture growth has been observed for bubbles bursting in water (Spiel 1992; Deane 2013; Poujol et al. 2021).

We also consider the decay of pressure amplitude $P$ during Helmholtz resonance. When the air in the neck of the aperture vibrates, $P$ decays with time as

$$
P=P_{0} \exp (-\beta t)
$$

where $\beta$ is the decay rate. Here we consider 2 mechanisms of decay (Kinsler et al. 2000). First is the decay from radiation $\left(\beta_{\mathrm{r}}\right)$, expressed as

$$
\beta_{\mathrm{r}}=\frac{\left(\pi R_{\mathrm{a}} f_{\mathrm{H}}\right)^{2}}{c l^{\prime}} .
$$

Second is the decay from viscous and thermal dissipation $\left(\beta_{\mathrm{vt}}\right)$ at the neck wall, expressed as

$$
\beta_{\mathrm{vt}}=\frac{1}{R_{\mathrm{a}}} \sqrt{\frac{\pi \eta_{\mathrm{a}} f_{\mathrm{H}}}{\rho_{\mathrm{a}}}}\left(1+\frac{\gamma_{\mathrm{h}}-1}{\sqrt{P r}}\right),
$$

where $\eta_{\mathrm{a}}$ is viscosity, $\rho_{\mathrm{a}}$ is density, and $\operatorname{Pr}=\eta_{\mathrm{a}} / \rho_{\mathrm{a}} \kappa_{\mathrm{a}}$ is the Prandtl number where $\kappa_{\mathrm{a}}$ is the thermal diffusivity. For our experiments (air), $\eta_{\mathrm{a}}=1.827 \times 10^{-5}$ Pas, $\rho_{\mathrm{a}}=1.193$ $\mathrm{kg} / \mathrm{m}^{3}$, and $P r=0.71$. Both $\beta_{\mathrm{r}}$ and $\beta_{\mathrm{vt}}$ depend on $R_{\mathrm{a}}$. Substituting Eq. (11) into Eqs.(13) and (14), and using $l^{\prime}=4 R_{\mathrm{a}} / \pi$, we obtain $\beta_{\mathrm{r}} \propto R_{\mathrm{a}}^{2} V^{-1}, \beta_{\mathrm{vt}} \propto R_{\mathrm{a}}^{-3 / 4} V^{-1 / 4}$. Thus, for a given $V$, as $R_{\mathrm{a}}$ grows, $\beta_{\mathrm{r}}$ becomes the dominant decay mechanism.

Fourth, when the bubble becomes vertically elongated and the bubble film bursts rapidly, resonance may be excited within the bubble cavity. Vidal et al. (2010) studied the situation in which a soap film enclosing a cavity (length $L$ ) bursts. They compared the bubble rupture time $\left(t_{\text {burst }}\right)$ to the airwave propagation time $\left(t_{\text {prop }}=2 L / c\right)$ and showed that the pressure amplitude of the cavity resonance becomes large when $t_{\text {burst }}<t_{\text {prop. }}$.

Fifth, the overpressurized air in the bubble eventually vents out, and the bubble deflates. Kobayashi et al. (2010) observed pressure fluctuation arising from such venting and called it an air flow.

\section{A regime diagram of bubble bursting and airwave excitation}

Figure 4a shows the 3 main styles of bubble bursting and airwave excitation revealed by our experiments. Figure $4 \mathrm{~b}$ is a regime diagram mapping the different styles in the parameter space of volumetric packing fraction $\phi$ of the suspensions and bubble volume $V$. Varying $\phi$ results in changing the rheology (Fig. 1). Here we injected 
(a)

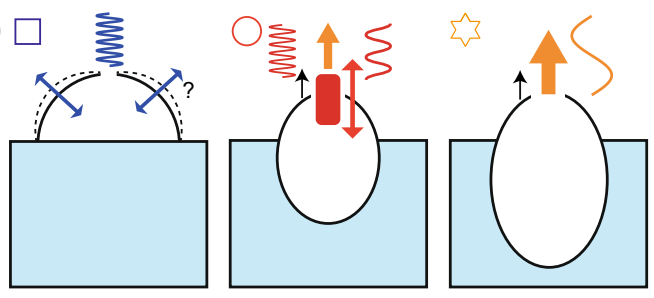

(b)
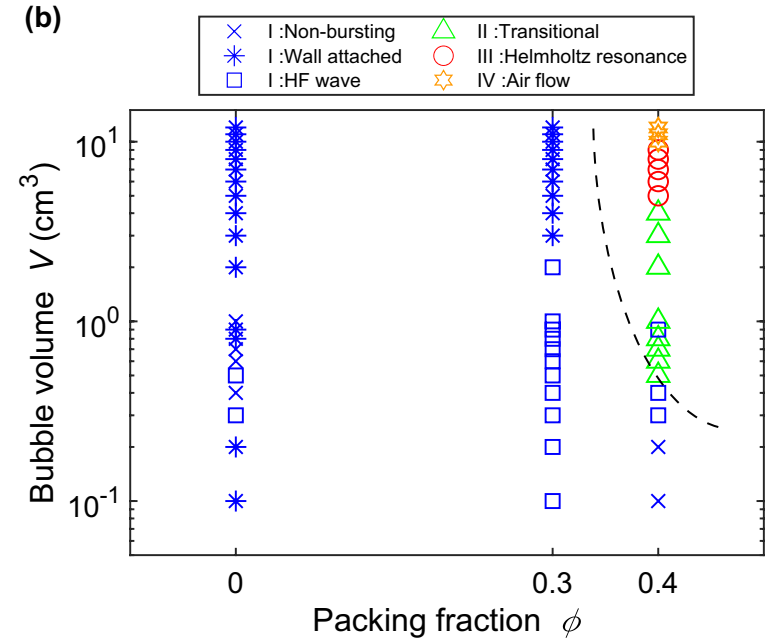

Fig. 4 a Schematic diagram showing the 3 main styles of bubble bursting and airwave excitation. Left : a hemispherical cap bubble sits at the surface and bursts, exciting a high-frequency (HF) wave. A "?" indicates that the modes are unknown. Middle : a bubble bursts while it is rising and excites Helmholtz resonance (mostly with HF wave component), and later vents an air flow. Right : a bubble bursts while it is rising and vents an air flow. $\mathbf{b}$ A regime diagram in the parameter space of suspension $\phi$ and bubble volume $V$. A broken curve indicates the main regime boundary (see text for details)

bubbles with 21 different $V\left(=0.1-12 \mathrm{~cm}^{3}\right)$ into suspensions with 3 different $\phi(=0,0.3,0.4)$. We conducted 5 runs per each combination of $V$ and $\phi$, and then classified the result into 8 cases, which were then grouped into the following 4 regimes (I-IV) ; I : non-bursting, wallattached, high-frequency (HF) wave, II : transitional, III : Helmholtz resonance, and IV : air flow. The details of these 8 cases and the method used to classify them into the 4 regimes are described in the Additional file 7: Material. In what follows, non-bursting and wall-attached cases are not analyzed further.

A key feature of Fig. $4 \mathrm{~b}$ is that the style of bubble bursting can be broadly classified into 2 types, whose boundary is indicated by a broken curve above which Helmholtz resonance is excited. For all runs using suspensions of $\phi=0,0.3$, and for runs using a suspension of $\phi=0.4$ with bubbles smaller than critical $\left(V \leq 0.4 \mathrm{~cm}^{3}\right)$, the bubble forms a hemispherical cap after surfacing and bursts thereafter, exciting a high-frequency (HF) wave (regime I). On the other hand, for runs using a suspension of $\phi=0.4$ and bubbles larger than critical $(V \geq 5$ $\mathrm{cm}^{3}$ ), the bubble bursts while it is rising when it partially protrudes above the surface and excites Helmholtz resonance (mostly with HF wave component) and/or air flow (Regimes III and IV). Regime II consists of a mixture of these 2 styles. In what follows, we describe the details of each of the styles shown in Fig. 4a.

\section{Diversity of bubble bursting and airwave excitation}

\section{A hemispherical cap bubble bursts and excites high-frequency wave}

Figure 5 shows a case in which a hemispherical cap bubble bursts and excites a high-frequency (HF) wave (case 3 in regime I). Here the bubble arrived at the surface, formed a cap, and after $\sim 25 \mathrm{~s}$ elapsed (i.e., the drainage time, see Additional file 7: Fig. S8), it burst, and the film disappeared within $\sim 1 \mathrm{~ms}$. Since Eo (Eq. (4)) of the injected bubble is $E o=71 \gg 1$, we infer that the bubble film thinned by gravitational drainage (Additional file 7 : Material).

In our experiments, an HF wave is defined as a wave with a peak frequency $f_{\mathrm{p}}>9200 \mathrm{~Hz}$ having an irregular waveform. A spectrogram indicates that the HF wave shown here has primary and secondary peaks at $\sim 14000$ $\mathrm{Hz}$ and $\sim 23000 \mathrm{~Hz}$, respectively, and these peaks are also evident from the power spectrum (Fig. 10). Figure $5 \mathrm{a}, \mathrm{b}$ shows that the timing of the aperture opening is within the time frame of $0.07<t<0.4 \mathrm{~ms}$, where the uncertainty arises from the camera exposure time $(0.33$ $\mathrm{ms})$. Since the travel time of the sound wave from the bubble to the microphone is $<0.07 \mathrm{~ms}$, we infer that a large pressure amplitude $(\sim 0.09 \mathrm{~Pa})$ at $t \sim 0.58 \mathrm{~ms}$ was excited after the bubble burst.

We further compared the timings of the airwave onset and bursting of 87 runs, which we classified as HF waves (case 3). We find that for all runs but one (98.9\%), the onset of the airwave was coincident or after the time frame that showed bursting. This result indicates that the HF wave is excited after bursting. It is also consistent with the result of Kobayashi et al. (2010), who showed that airwave is excited after bursting when a $V=8,32$ $\mathrm{cm}^{3}$ bubble ascends and bursts in a syrup solution with a viscosity of $\eta \geq 0.17$ Pas, where $V$ and $\eta$ are comparable to those in our experiments.

\section{A rising bubble bursts and excites Helmholtz resonance}

Figure 6 shows a case in which a bubble excites Helmholtz resonance (case 5 in regime II). In contrast to the case shown in Fig. 5, the bubble bursts while it is rising and when it partially protrudes above the surface, i.e., 
(a)

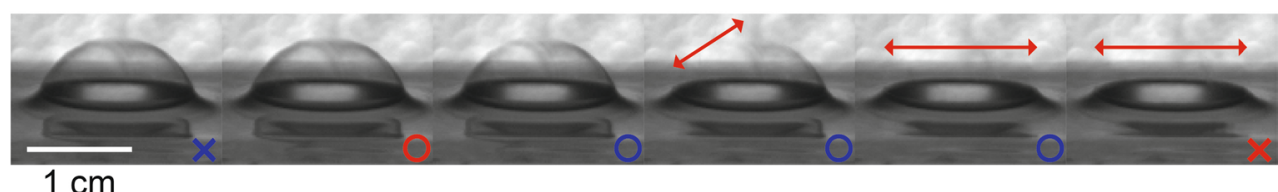

$1 \mathrm{~cm}$

(b)

b)

(c)
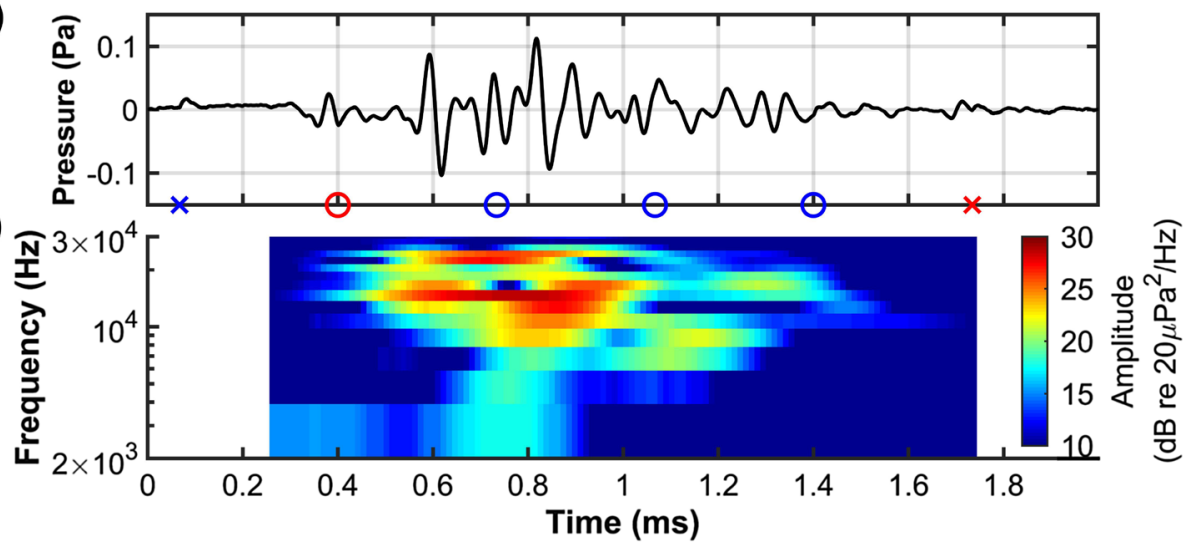

Fig. 5 An example of HF wave (case 3). $V=0.6 \mathrm{~cm}^{3}$ hemispherical cap bubble bursts in a suspension of $\phi=0.3$. a Time-lapse images (see Additional file 1: Movie 1). Blue $x$ : before bursting, $\bigcirc$ : after bursting, red $x$ : film disappearance. Red arrows indicate the aperture. $\mathbf{b}$ Waveform (detrended, highpass filtered at $800 \mathrm{~Hz}$ ). Markers correspond to the timing shown in (a) and are plotted at the end of the exposure time of each image. c Spectrogram of (b) using a 0.512 ms sliding window and a 0.5 ms overlap

before it forms a hemispherical cap (see also Additional file 2: Movie 2). Here a dimple forms at the top of the bubble before it bursts. After bursting the aperture grows and the bubble deflates. The waveform shows a damped oscillation with a peak frequency of $f_{\mathrm{p}} \simeq 4360 \mathrm{~Hz}$, which decays in about $4 \mathrm{~ms}$. This waveform, characterized by a lower frequency that continues for a longer time, is clearly distinguishable from that of the HF wave (Fig. 5b). The onset of the airwave coincides with the timing of the aperture opening within the camera frame rate $(0.5 \mathrm{~ms})$. The spectrogram (Fig. 6c) shows that the peak frequency glides toward a higher frequency. It also shows that an HF wave component of $f_{\mathrm{p}} \sim 21270 \mathrm{~Hz}$ is excited. We calculate $f_{\mathrm{H}}$ (Eq. (11)) using the aperture radius $R_{\mathrm{a}}$ measured from the images and plot on the spectrogram. The measured $f_{\mathrm{p}}$ agrees well with the calculated $f_{\mathrm{H}}$, from which we interpret that frequency gliding is a consequence of aperture growth (see below Eq. (11) for an explanation). We highpass and bandpass filter the waveform to separate the signals arising from the HF wave component and Helmholtz resonance, respectively, which we show in Fig. $6 \mathrm{~d}$. The onset and duration of these 2 waves are close to each other.

Figure 6e shows the waveforms of both Mic 1 (lower) and Mic 2 (upper) covering a longer time span (70 ms). The waveform of the Helmholtz resonance recorded by Mic 2 is delayed relative to that recorded by Mic 1 by $0.147 \mathrm{~ms}$, and its amplitude is smaller. The measured time delay is close to the estimated $0.145 \mathrm{~ms}(=5 \mathrm{~cm} / c)$ confirming that this signal is a sound wave. The waveform of Mic 1 shows that a low-frequency (period $\sim 20 \mathrm{~ms}$ ) signal follows after the Helmholtz resonance. This signal has an amplitude which exceeds the noise $(\leq 0.02 \mathrm{~Pa})$ and is not observed by Mic 2 further away from the bubble. Since these results are reproducible (Additional file 7: Fig. S7), we interpret this signal as an air flow vented by the deflating bubble (Kobayashi et al. 2010). We calculate the lower limit air flow velocity $v_{\mathrm{af}}$, assuming that the air flow was vented immediately after the Helmholtz resonance decayed. Using the time lag and the distance between the bubble and Mic 1, we obtain $v_{\mathrm{af}} \sim 1.6 \mathrm{~m} / \mathrm{s}$. The overpressure $\Delta P$ needed to generate this air flow can then be estimated as $\Delta P \sim \rho_{\mathrm{a}} v_{\mathrm{af}}^{2} / 2 \sim 1.5 \mathrm{~Pa}$, which is of the same order of magnitude as $\Delta P \sim 2 \sigma / R_{\mathrm{b}}=6.5 \mathrm{~Pa}$. We note that an air flow must always exist, since overpressurized air in the bubble would always flow out after the bubble bursts. However, the waveform we identified as an air flow is detected only in limited cases. We also note that the amplitude of the waveform will be affected by the location of aperture opening, which differs among the runs. Compared to Fig. 5, the bubble aperture grows slowly and it takes about $\sim 36.5 \mathrm{~ms}$ after the aperture opens for the bubble film to disappear, which is indicated by $x$ in Fig. 6e. This experiment demonstrates that bubble bursting can simultaneously excite a Helmholtz resonance $\left(\mathcal{O}\left(10^{3}\right) \mathrm{Hz}\right)$, an $\mathrm{HF}$ wave component $\left(\mathcal{O}\left(10^{4}\right) \mathrm{Hz}\right)$, 
(a)

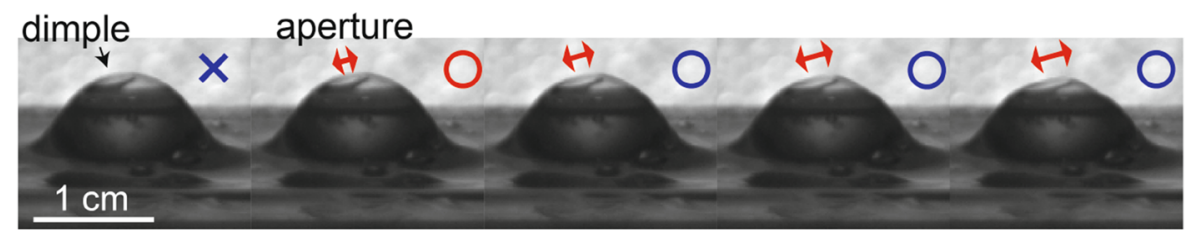

(b)

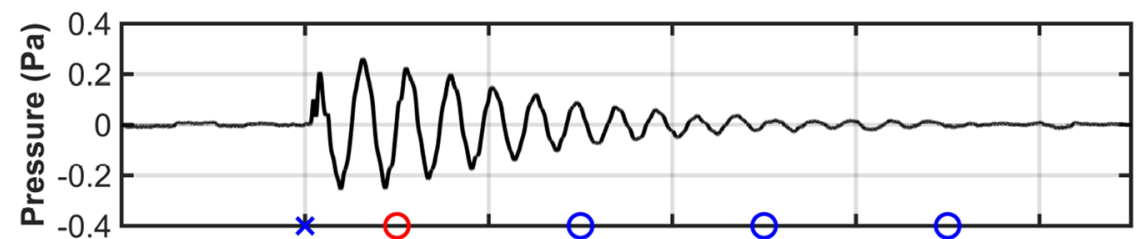

(c)
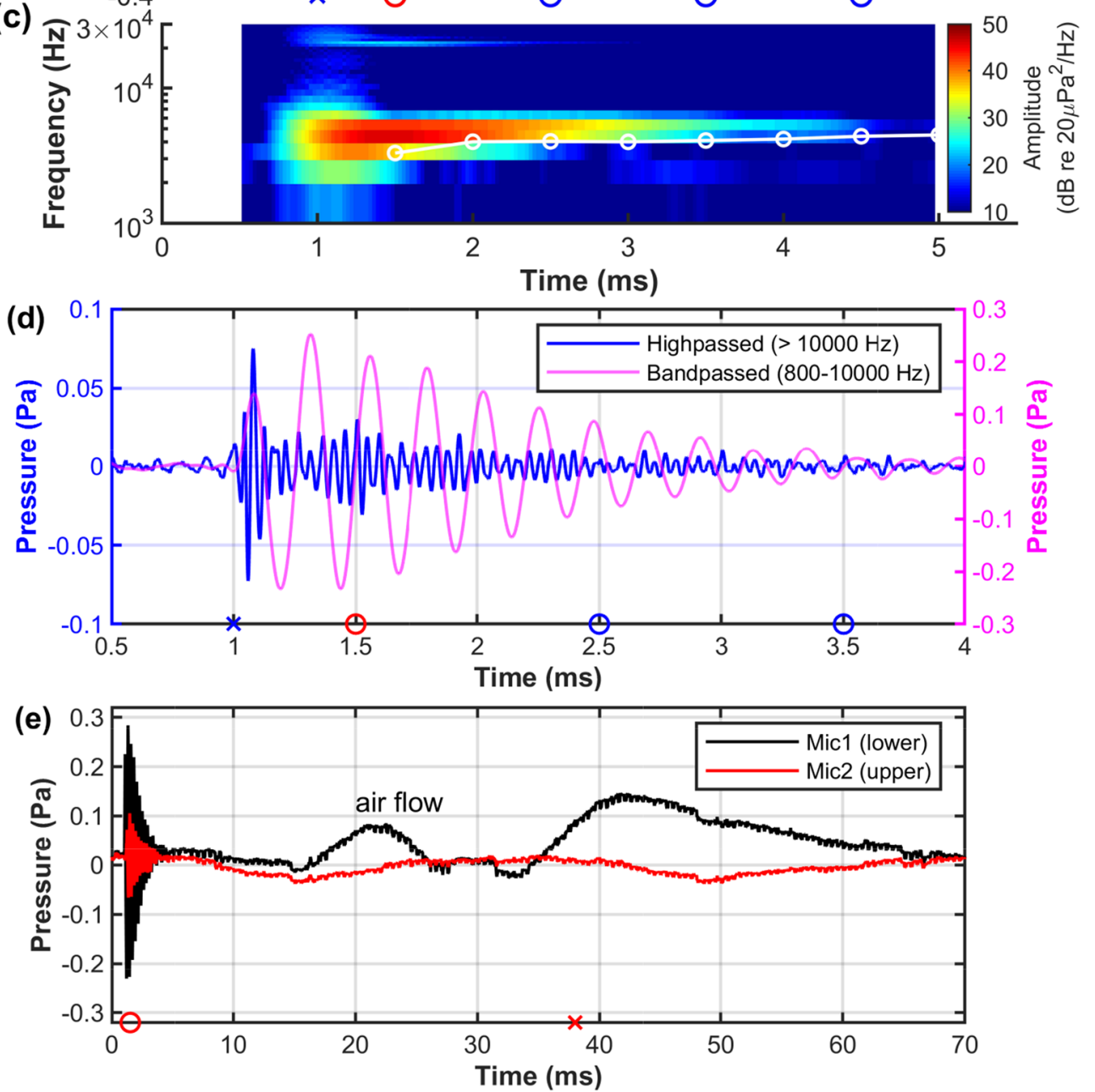

Fig. 6 An example of Helmholtz resonance (small bubble, case 5). $V=0.6 \mathrm{~cm}^{3}$ bubble bursts while it is rising in a suspension of $\phi=0.4$. a Time-lapse images (see Additional file 2: Movie 2) $\times$ and $\bigcirc$ indicate before and after aperture opening, respectively. $\mathbf{b}$ Waveform (detrended, highpass filtered at $>800 \mathrm{~Hz}$ ). c Spectrogram of (b) using a $1.024 \mathrm{~ms}$ sliding window and a $1 \mathrm{~ms}$ overlap. $\bigcirc$ indicates $f_{\mathrm{H}}$ calculated from Eq. (11) using measured $R_{\mathrm{a}}$. $\mathbf{d}$ High- ( $>10000 \mathrm{~Hz}$, left axis) and bandpass ( $800-10000 \mathrm{~Hz}$, right axis) filtered result of (b) shown for a shorter time span. e Waveform shown for a longer time span (running-averaged raw data) indicating Helmholtz resonance followed by a low-frequency air flow. A red $x$ indicates the time of film disappearance 
(a)

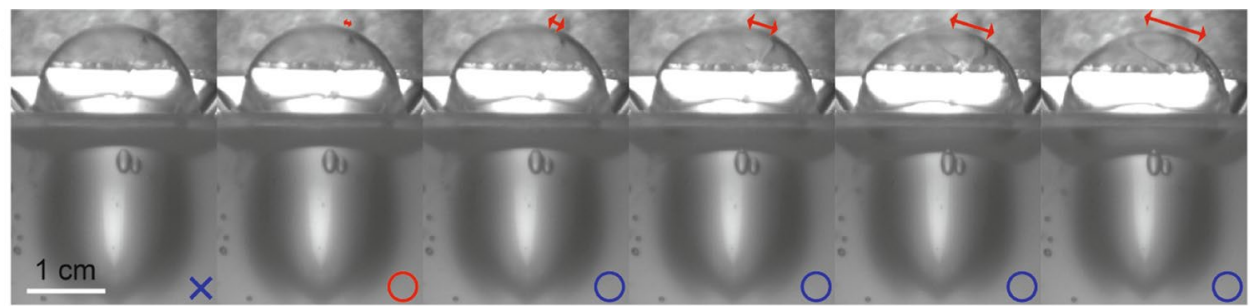

(b)

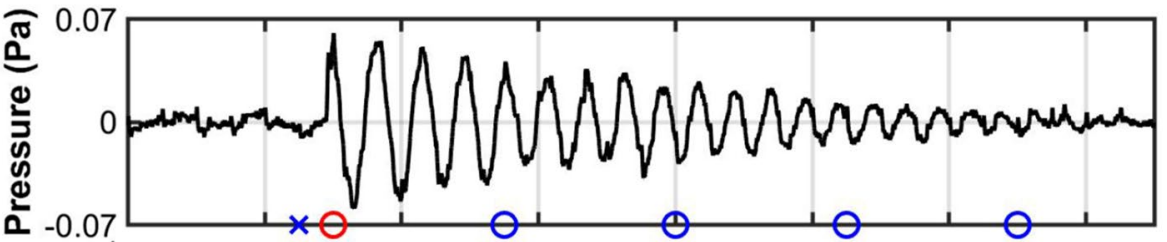

(c)

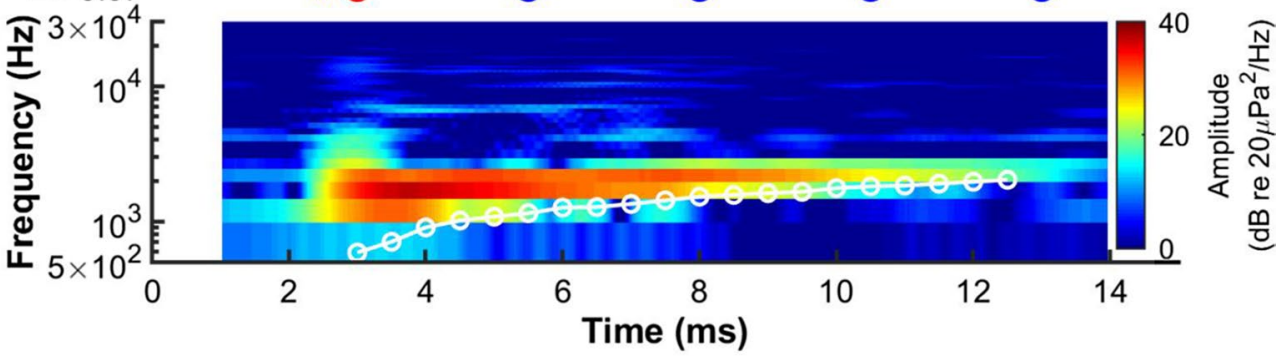

Fig. 7 An example of Helmholtz resonance (large bubble, case 5). $V=7 \mathrm{~cm}^{3}$ bubble bursts while it is rising in a suspension of $\phi=0.4$. a Time-lapse images (see Additional file 3: Movie 3). b Waveform (detrended, highpass filtered at $800 \mathrm{~Hz}$ ). c Spectrogram of (b) using a $2.048 \mathrm{~ms}$ sliding window and 2.030 ms overlap. $O$ indicates the $f_{\mathrm{H}}$ calculated from Eq. (11) using measured $R_{a}$

and an air flow $(\mathcal{O}(10) \mathrm{Hz})$, covering a broad frequency band.

Figure 7 shows another example of Helmholtz resonance (case 5 in regime III). Here the bubble has a larger volume $\left(V=7 \mathrm{~cm}^{3}\right)$ than that shown in Fig. 6 and becomes vertically elongated to form a slug. The bubble bursts while it is rising and the waveform indicates damped oscillation. The calculated $f_{\mathrm{H}}$ plotted on the spectrogram is initially smaller than the measured peak frequency $f_{\mathrm{p}}$ but the agreement improves as the aperture grows, confirming that Helmholtz resonance is excited. There are at least 2 explanations for $f_{\mathrm{H}}$ being initially smaller than $f_{\mathrm{p}}$. The first is that the aperture of this run is an ellipse, and the measured $R_{\mathrm{a}}$ corresponds to its minor axis. The second is that the expression of estimated neck length $l^{\prime}$ has a range (see below Eq. (11) for explanations), and may have been overestimated. Compared to Fig. 6c, the peak frequency $\left(f_{\mathrm{p}} \sim 1670 \mathrm{~Hz}\right)$ is smaller and continues longer $(\sim 10 \mathrm{~ms})$. The spectrogram also shows simultaneous excitation of an HF wave component with a frequency of $f_{\mathrm{p}} \sim 6500 \mathrm{~Hz}$, which is lower than that shown in Fig. 6c.

Figure 8 is a further example of Helmholtz resonance excited by a rising bubble (case $4 \mathrm{~b}$ in regime II). Here a thin film forms at the apex of the bubble, and when it bursts, both the Helmholtz resonance $(\sim 3000 \mathrm{~Hz})$ and an HF wave component in the range of $\sim 1-3 \times 10^{4} \mathrm{~Hz}$ are excited and these are superimposed on the waveform. The measured $f_{\mathrm{p}}$ agrees well with the calculated $f_{\mathrm{H}}$ plotted on the spectrogram. The higher $f_{\mathrm{p}}$ than that shown in Fig. 7 despite a similar $V$ can be explained as a result of a larger $R_{\mathrm{a}}$. When the thin film bursts, the aperture opens quickly at a velocity comparable to that in the case where a hemispherical cap bubble bursts and excites an HF wave.

Here we consider the possibility of cavity resonance within the bubble. The cavity resonance frequencies of the the runs shown in Figs. 7 and 8 are also $\mathcal{O}\left(10^{3}\right) \mathrm{Hz}$ (Additional file 7: Material). However Helmholtz resonance better explains the measured $f_{\mathrm{p}}$, its dependence on $R_{\mathrm{a}}$, and the glide toward a higher frequency. In our experiments, bubble rupture was slow and the cavity was short such that $t_{\text {burst }}>t_{\text {prop }}$, from which we infer that the excitation of cavity resonance was inefficient (Vidal et al. 2010).

\section{A rising bubble bursts and vents an air flow}

Figure 9 shows an example of an air flow dominant case (case 6a in regime IV). As in Figs.6, 7, 8, the bubble bursts when it partially protrudes above the surface. However 

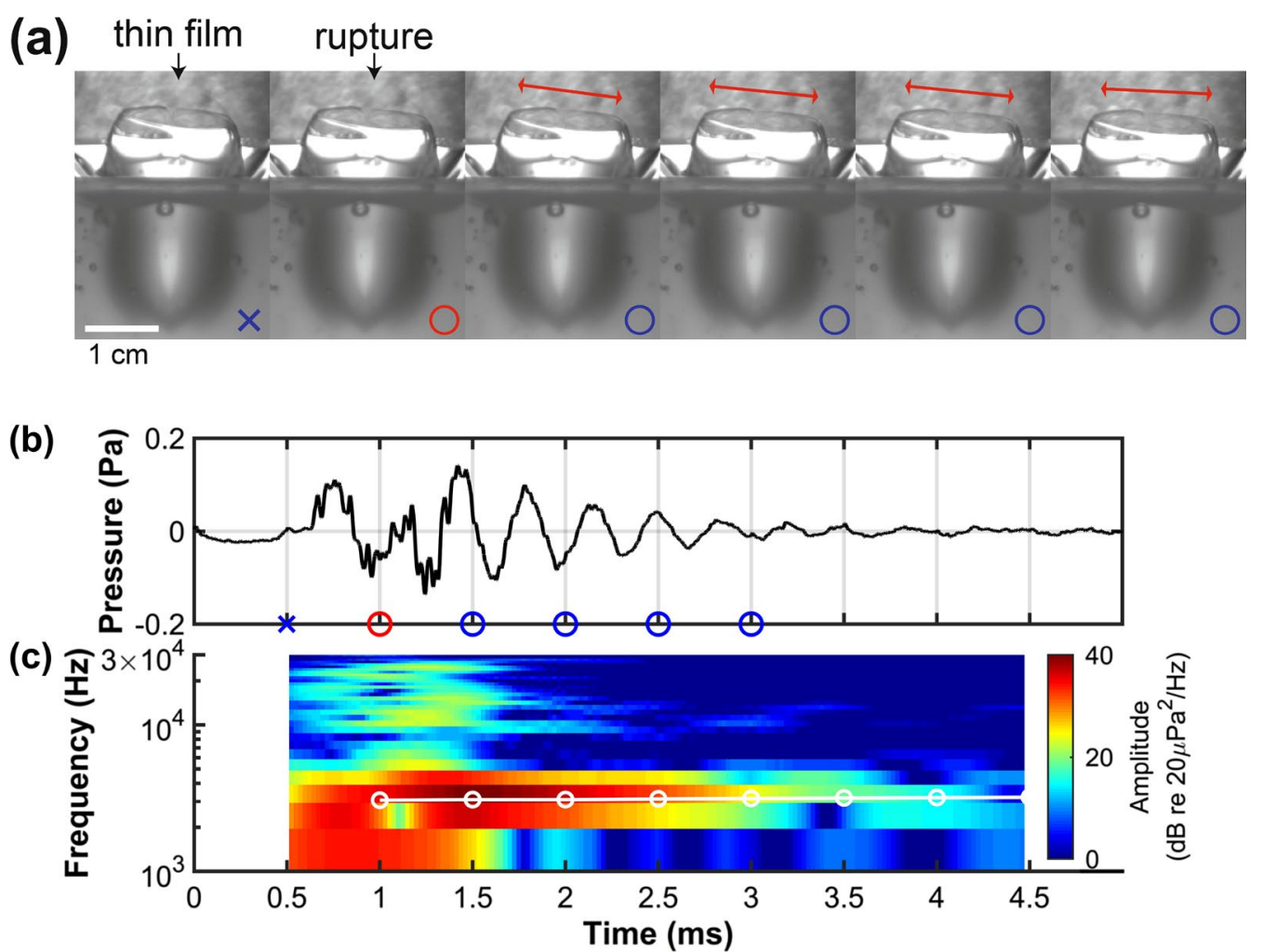

Fig. 8 An example of Helmholtz resonance excited after a thin film bursts (case $4 b$ ). $V=6 \mathrm{~cm}^{3}$ bubble bursts while it is rising in a suspension of $\phi=0.4$. a Time-lapse images (see Additional file 4: Movie 4). Red arrows indicate the aperture. $\mathbf{b}$ Waveform (detrended, highpass filtered at $800 \mathrm{~Hz}$ ). c Spectrogram of (b) using a $1.024 \mathrm{~ms}$ sliding window and a $1.000 \mathrm{~ms}$ overlap. $\bigcirc$ indicates the $f_{\mathrm{H}}$ calculated using Eq. (11) and measured $R_{\mathrm{a}}$

different from Fig. 7, the amplitude of the waveform is dominated by an air flow, which continues for $\sim 100 \mathrm{~ms}$. In the spectrogram (Fig. 9c), we identify a weak signal (indicated by an arrow) at $f \sim 1400 \mathrm{~Hz}$ superimposed on the main air flow signal at $\mathcal{O}(10) \mathrm{Hz}$. The signal is excited immediately after the bubble bursts, and its frequency increases with time. The calculated Helmholtz resonance frequency $f_{\mathrm{H}}$ plotted on the spectrogram (see legend for the values of $f_{\mathrm{H}}$ ) bound the observed frequency from which we conclude that Helmholtz resonance was also excited.

\section{Comparison of power spectra}

Figure 10 compares the power spectra of 5 waveforms, which we showed in Figs.5, 6, 7, 8, 9. Here we highlight the characteristics of the spectra and their $V$ dependences.

First, when a hemispherical cap bubble excites an HF wave ( $\phi=0.3, V=0.6 \mathrm{~cm}^{3}$ case, Fig. 5), the power spectrum consists of 2 main peaks at $f_{\mathrm{p}}>10000 \mathrm{~Hz}$. Next when a rising bubble excites Helmholtz resonance $\left(\phi=0.4, V=0.6,6,7 \mathrm{~cm}^{3}\right.$, Figs.6, 7, 8), $f_{\mathrm{p}}$ values are $\mathcal{O}\left(10^{3}\right) \mathrm{Hz}$, and the spectra show that there is a trend of $f_{\mathrm{p}}$ decreasing with $V$. There is also a trend of the $f_{\mathrm{p}}$ of HF wave component decreasing with $V$. The $V$ dependence of $f_{\mathrm{p}}$ obtained using all available data and comparison with scaling laws will be described later. Finally, when a rising bubble excites an air flow $(\phi=0.4, V=12$ $\mathrm{cm}^{3}$ case; Fig. 9), the power of the Helmholtz resonance becomes much smaller but can still be identified on the spectrum.

\section{Aperture growth and estimates of film thickness}

High-speed images indicate that the aperture growth velocity $v_{\mathrm{a}}=d R_{\mathrm{a}}(t) / d t$ is slower when Helmholtz resonance is dominantly excited (Figs.6, 7) compared to other cases (Figs.5, 8, 9). To quantify the difference in $v_{\mathrm{a}}$, we measured the aperture radius $R_{\mathrm{a}}(t)$ as a function of time, which we show in Fig. 11a. The figure indeed shows that when the aperture opens slowly, Helmholtz resonance is dominantly excited, whereas when the aperture opens rapidly, the HF wave or air flow becomes dominant. We also measured the bubble surface radius $R_{\mathrm{b}}$ at $t=0.5 \mathrm{~ms}$, which we use to normalize $R_{\mathrm{a}}$ and the result is shown in Fig. 11b. The figure shows that the aperture grows rapidly to become $R_{\mathrm{a}} / R_{\mathrm{b}} \sim 1$ when the HF wave is dominant. 
(a)
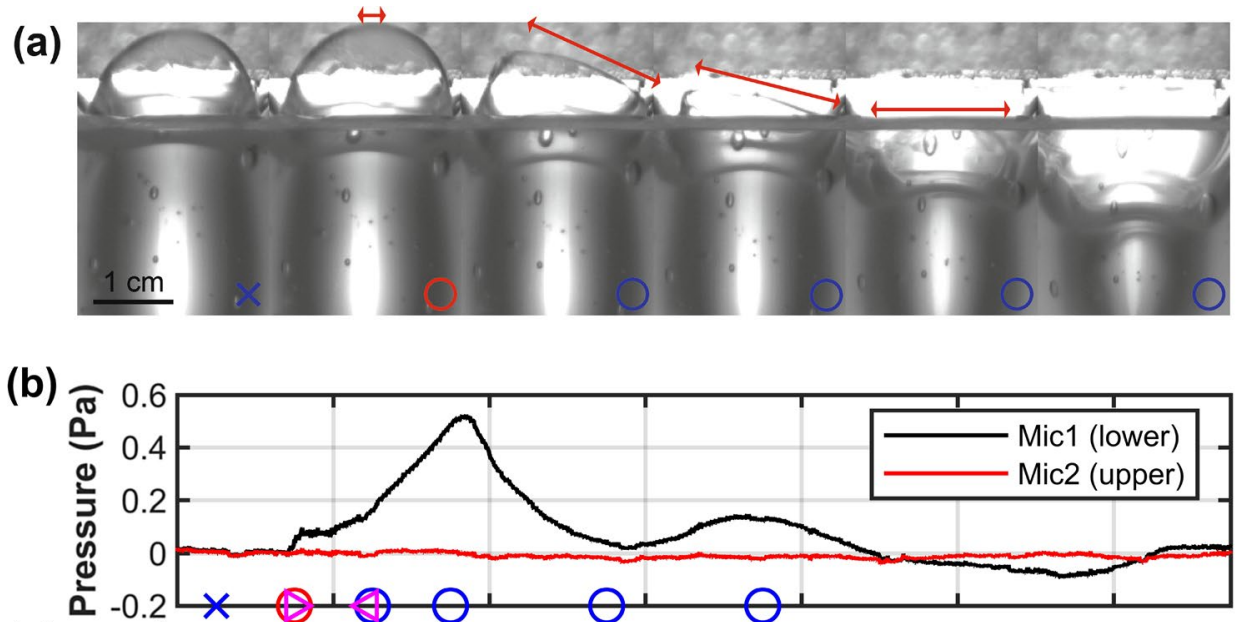

(c)

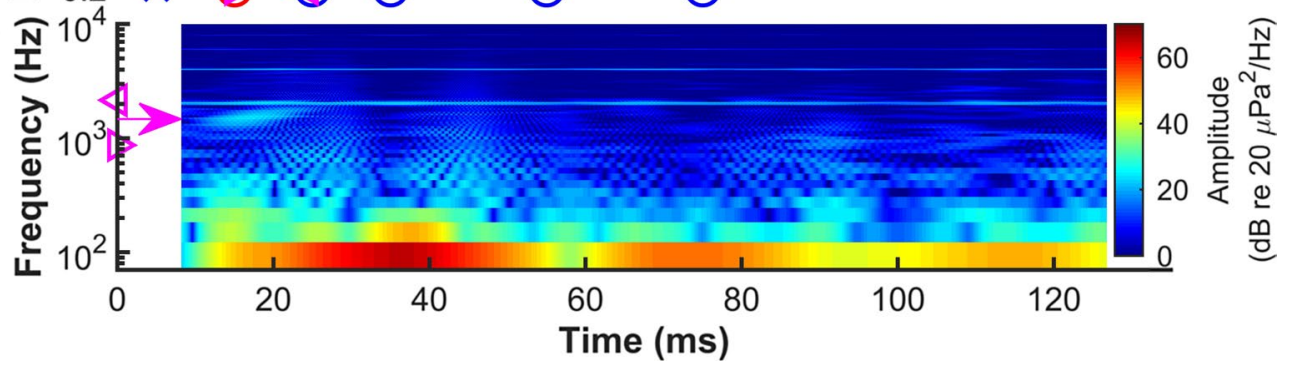

Fig. 9 An example of an air flow (case $6 a) . V=12 \mathrm{~cm}^{3}$ bubble bursts while it is rising in a suspension of $\phi=0.4$. a Time-lapse images (see Additional file 5: Movie 5). b Waveform (raw data) of the airwave recorded by 2 microphones. $\times$ and $\bigcirc$ correspond to the times indicated in (a). The pink $\triangleright$ and $\triangleleft$ indicate the times when the aperture radius $R_{a}$ was measured. c Spectrogram (time window $16.384 \mathrm{~ms}$, overlap $16.3 \mathrm{~ms}$ ) of Mic 1 (lower) data shown in (b). $\triangleright$ and $\triangleleft$ indicate $f_{H}=870$ and $2161 \mathrm{~Hz}$, calculated from Eq. (11) using $R_{a}$ measured at the respective times shown in (b). The pink arrow indicates the Helmholtz resonance excited after bursting

Following the method of Kobayashi et al. (2010), we use 2 models of aperture growth velocity to estimate the film thickness $h$ using the $R_{\mathrm{a}}(t)$ measurements shown in Fig. 11a. First is the inviscid model (Taylor 1959; Culick 1960), in which the aperture grows under the balance of capillary and inertial stresses at a constant velocity $v_{\mathrm{i}}$ and is expressed as

$$
v_{\mathrm{i}}=\sqrt{\frac{2 \sigma}{\rho h}} .
$$

Second is the viscous model (Debrégeas et al. 1998), in which the aperture grows under the balance of capillary and viscous stresses at a velocity $v_{\mathrm{v}}$ which accelerates as $R_{\mathrm{a}}(t)$ grows, and is expressed as

$$
v_{\mathrm{v}}=\frac{\sigma}{\eta h} R_{\mathrm{a}}(t)
$$

such that $R_{\mathrm{a}}(t)$ grows as

$$
R_{\mathrm{a}}(t)=R_{0} \exp \left(\frac{\sigma}{\eta h} t\right) .
$$

These 2 models are applicable during the initial stage of aperture growth when we may approximate that the film near the aperture is horizontal and $h$ is constant.

To estimate $h$, we fit our $R_{\mathrm{a}}(t)$ measurements using the 2 models (see Additional file 7: Fig. S9 for fitted examples), using the time span $\Delta t$ which maximizes the correlation coefficient $r$. We did not fit $R_{\mathrm{a}}(t)$ for cases $4 \mathrm{a}$ and $4 \mathrm{~b}$ because we could not time-resolve the initial stage of rapid aperture growth. $\eta$ in Eq. (17) is estimated as follows. The strain rate $\dot{\gamma}$ of the aperture growth is $\dot{\gamma} \sim v_{\mathrm{a}} / R_{\mathrm{a}} \sim \mathcal{O}\left(10^{2}-10^{4}\right) 1 / \mathrm{s}$ (Debrégeas et al. 1998), where we used the measured $v_{\mathrm{a}} \sim \mathcal{O}(0.1-10) \mathrm{m} / \mathrm{s}$. Our viscosity $\eta$ measurements (Fig. 1b) indicate that at the high $\dot{\gamma}$ limit, $\eta$ approaches the value estimated from Eq. (2), from which we estimate $\eta=0.9$ Pas for a suspension of $\phi=0.4$. $h$ thus calculated from the 2 models are summarized in Table 1. 


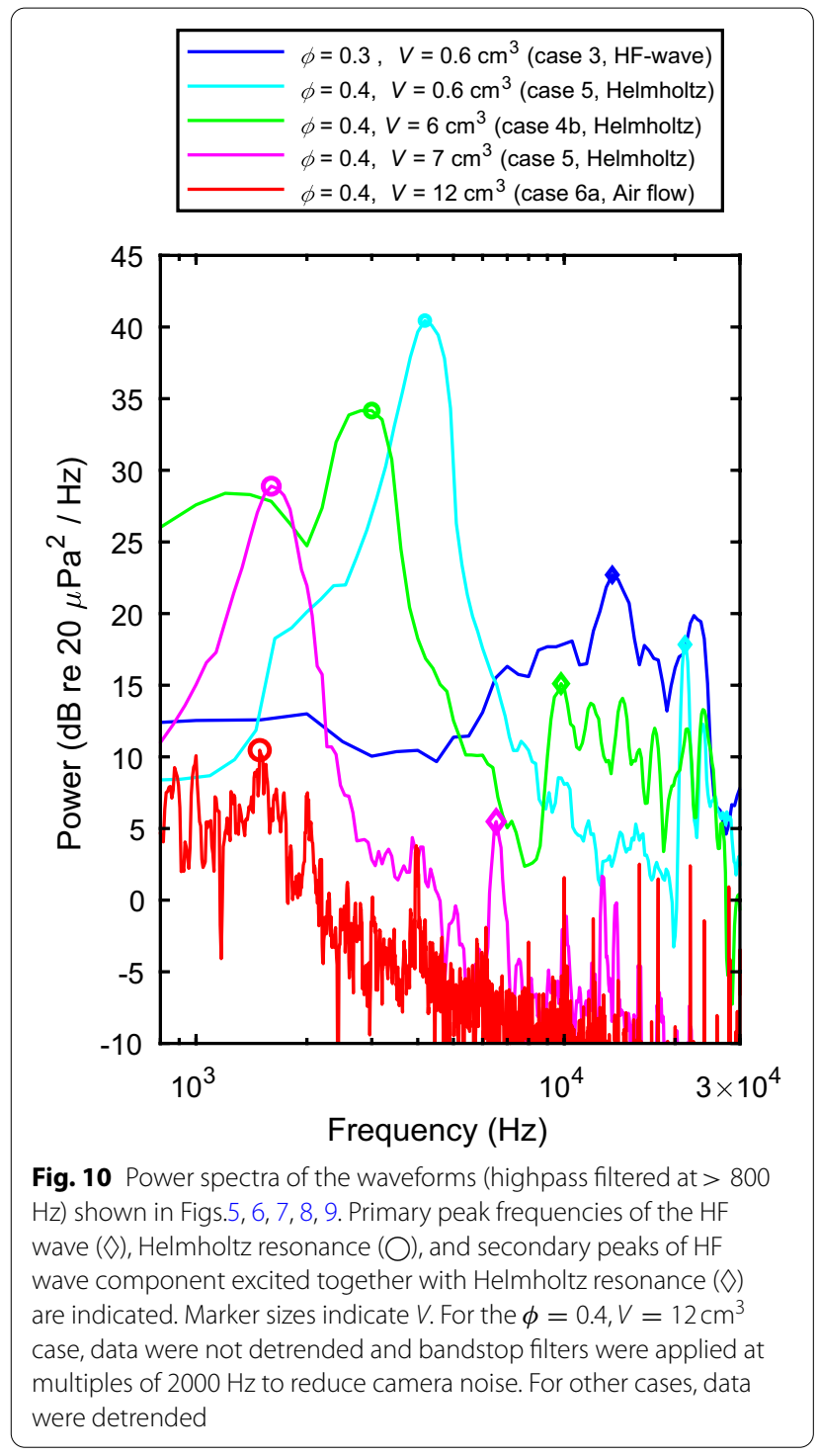

Our results indicate that $h=\mathcal{O}(1-10) \mu \mathrm{m}$ for both models and that the inviscid model gives a better fit (larger $r$ and a longer $\Delta t$ ). This result is consistent with those of Kobayashi et al. (2010), who obtained the $h$ of bubbles bursting in a syrup solution with a comparable viscosity of $\eta \simeq 1.9$ Pas. The values in Table 1 indicate that the $h$ estimated for cases 5 and 6 a (Helmholtz resonance) are thicker than those estimated for cases 3 (HF wave) and $6 \mathrm{~b}$ (air flow) as a result of smaller $v_{\mathrm{a}}$. We note that the estimated $h$ is comparable to or larger than the particle diameter $\left(d_{\mathrm{p}} \simeq 2 \mu \mathrm{m}\right)$ suspended in the liquid. From the viscous model, we obtained $h / d_{\mathrm{p}} \sim 11-22$. This model uses suspension viscosity $\eta$, which assumes a continuum. However $h / d_{\mathrm{p}}=\mathcal{O}(10)$ suggests that this

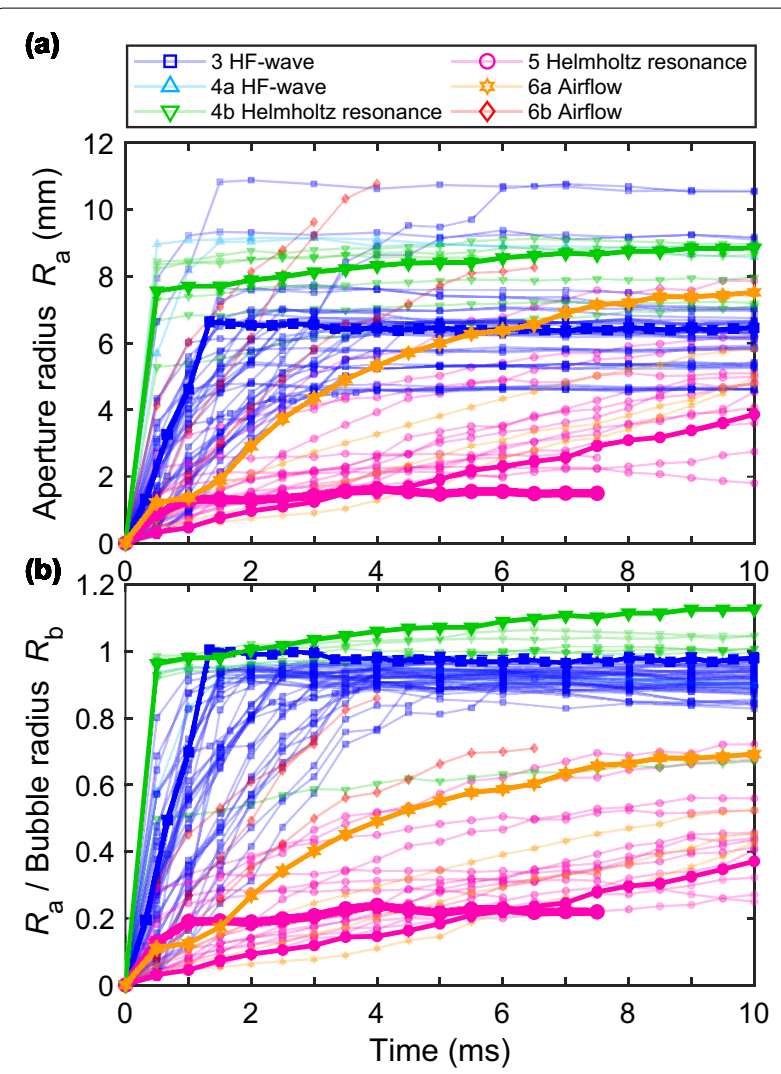

Fig. 11 Aperture radius vs. time. a $R_{\mathrm{a}}(t)$ vs. time. Thick lines indicate the $R_{\mathrm{a}}(t)$ of the runs shown in Figs.5, 6, 7, 8, 9 (the thickest pink line indicates the $R_{a}(t)$ for Fig. 6). Thin lines indicate runs using suspensions of $\phi=0.4$, where we used all runs whose timings of aperture opening could be determined accurately. Ranges of $V\left(\mathrm{~cm}^{3}\right)$ are as follows; case $3: 0.3-3$; case $4 a: 2$ - 4; case $4 b: 3$ - 6; case $5: 0.6$ - 7; case 6a : 10 - 12; case 6b: 10 - 12. b Normalized $\left(R_{a} / R_{b}\right)$ version of (a) (see text for details)

Table 1 Film thickness $h$ (standard deviation $\delta h$ ) calculated by fitting $R_{\mathrm{a}}(t)$ ( $\phi=0.4$ suspension) to inviscid and viscous models. $\mathrm{HF}$ : high-frequency wave; $\mathrm{H}$ : Helmholtz resonance; $\mathrm{AF}$ : air flow; $N$ : number of runs used; $r$ : correlation coefficient (standard deviation $\delta r$ ); $\Delta t$ : Time span used for the fit. In terms of $N$, the inviscid model gives a higher $r$ for $69 \%$ of the runs

\begin{tabular}{llll}
\hline Case & $\boldsymbol{N}$ & Inviscid $\boldsymbol{h}(\boldsymbol{\delta} \boldsymbol{h})(\boldsymbol{\mu} \mathbf{m})$ & Viscous $\boldsymbol{h}(\boldsymbol{\delta} \boldsymbol{h})(\boldsymbol{\mu} \mathbf{m})$ \\
\hline $3(\mathrm{HF})$ & 35 & $5(4)$ & $26(21)$ \\
$5(\mathrm{H})$ & 16 & $69(93)$ & $44(36)$ \\
$6 \mathrm{a}(\mathrm{AF}+\mathrm{H})$ & 4 & $65(60)$ & $36(25)$ \\
$6 \mathrm{~b}(\mathrm{AF})$ & 3 & $4(4)$ & $22(12)$ \\
Total, $r(\delta r)$ & 58 & $0.984(0.002)$ & $0.969(0.003)$ \\
Total, $\Delta t(\mathrm{~ms})$ & 58 & $2.4 \pm 2.4$ & $1.2 \pm 1.0$ \\
\hline
\end{tabular}




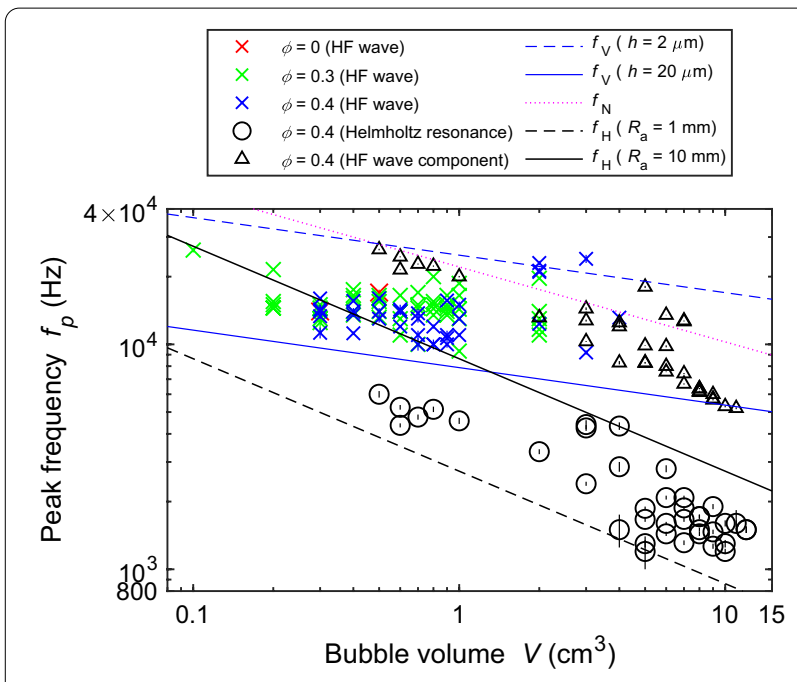

Fig. 12 Bubble volume $V$ dependence of the peak frequency $f_{p}$ (with errors) of the airwaves. An HF wave is excited by a hemispherical cap bubble. Helmholtz resonance and the HF wave component are excited together by a bubble which bursts when it partially protrudes above the surface. $f_{V}, f_{N}$, and $f_{H}$ are calculated from Eqs.(9), (10), and (11) respectively, using $h$ and $R_{a}$ values given in the legend (see text for details). A hemispherical cap bubble was assumed to calculate $f_{v}$, $f_{\mathrm{N}}$. For a partially protruding bubble with $/ / R_{\mathrm{b}}=1$ (Eq. (7)), $f_{\mathrm{V}}$ becomes smaller by a factor of $\sim 0.8$. $\rho$ of $\phi=0.4$ suspension was used to calculate $f_{v}$

assumption may have been only marginally valid. Here we assumed that the 2 models are applicable. Their applicability need to be further checked using direct measurement of $h$ and a sufficiently time-resolved high-speed imagery.

\section{Bubble volume dependence of peak frequency and decay}

We determined the primary peak frequency $f_{\mathrm{p}}$ of the HF wave and Helmholtz resonance as follows. First we applied a highpass filter $(>800 \mathrm{~Hz})$ to remove the lowfrequency air flow and bandstop filters $(2000,4000 \mathrm{~Hz}$, or $3000,6000 \mathrm{~Hz}$ ) to reduce camera noise arising from frame rate. We did not use 2 runs from case 3 (HF wave) because the amplitude was small $(<0.02 \mathrm{~Pa})$ near the noise level, and 4 runs (of which 3 had an amplitude $<0.02 \mathrm{~Pa}$ ) from case $6 \mathrm{~b}$ (air flow dominant) because they did not excite an HF wave or Helmholtz resonance. We then calculated the power spectrum of each waveform and determined the $f_{\mathrm{p}}$. When the primary $f_{\mathrm{p}}$ corresponded to the Helmholtz resonance, we also determined the secondary $f_{\mathrm{p}}$, which corresponds to the HF wave component (e.g., Figs.6, 7, 8). In order to determine the secondary $f_{\mathrm{p}}$, we smoothed the power spectrum by a 7 point running average, and searched for the peak in the frequency range above the primary $f_{\mathrm{p}}$ and above 5000 $\mathrm{Hz}$.

The $f_{\mathrm{p}}$ values thus determined are plotted as a function of $V$ in Fig. 12. Each $\times$ (red, green, blue) indicates the $f_{\mathrm{p}}$ of the HF wave, scattered over a range of 9200 $26250 \mathrm{~Hz}$, and they are poorly correlated with $V$ (correlation coefficient $r=-0.103$ ). $\bigcirc$ and $\triangle$ (black) indicate the $f_{\mathrm{p}}$ of the Helmholtz resonance and HF wave component, respectively, both of which decrease with $V$ (negative correlation of $r=-0.864$ and -0.878 , respectively). A possible reason for the different $r$ for the HF wave and HF wave component will be explained in the next section.

We calculated the frequencies excited by the film vibration $f_{\mathrm{V}}, \mathrm{N}$-wave $f_{\mathrm{N}}$, and Helmholtz resonance $f_{\mathrm{H}}$, which we show in Fig. 12. We assumed $h$ in the range of $h=2-20 \mu \mathrm{m}$ to calculate $f_{\mathrm{V}}$, such that $f_{\mathrm{V}}$ bound most of the measured $f_{\mathrm{p}}$ of the HF wave. These $h$ values are comparable to the $h \simeq 5, \simeq 26 \mu \mathrm{m}$ (case 3 , HF wave) estimated from aperture growth velocity (Table 1). Similarly, we assumed an aperture radius $R_{\mathrm{a}}$ in the range of $R_{\mathrm{a}}=1-10 \mathrm{~mm}$, to calculate $f_{\mathrm{H}}$, such that $f_{\mathrm{H}}$ bound the measured $f_{\mathrm{p}}$. These $R_{\mathrm{a}}$ are consistent with the measured $R_{\mathrm{a}}$ (Fig. 11a).

The figure shows that the measured $f_{\mathrm{p}}$ of the HF wave and Helmholtz resonance can be explained by $f_{\mathrm{V}}$ and $f_{\mathrm{H}}$, respectively, using reasonable values of $h$ and $R_{\mathrm{a}}$. The scatter of $f_{\mathrm{p}}$ for the same $V$ can be attributed to the variation of $h$ or $R_{\mathrm{a}}$. We note that the power spectra of the HF wave and HF wave component (Fig. 10) have multiple peaks with comparable power, which may also be the reason for the scatter. We add that $f_{\mathrm{N}}$ can also become a candidate to explain the HF wave.

When Helmholtz resonance is excited, we may use the decay rate $\beta$ to infer the damping mechanism. Figure 13a shows an example of such analyses. We fit the envelope of the waveform to an exponential decay function (Eq. (12)) and obtain $\beta$. We calculated $\beta$ for all runs that excited Helmholtz resonance (cases $4 \mathrm{~b}$, $5,6 \mathrm{a})$ and Fig. 13b shows the obtained $\beta$ plotted vs. peak frequency $f_{\mathrm{p}}$. Here $f_{\mathrm{p}}$ is obtained using the same method we used in Fig. 12. The figure shows that there is a trend of $\beta$ increasing with $f_{\mathrm{p}}$. There are 2 outliers at $f_{\mathrm{p}}=1200$ and $1500 \mathrm{~Hz}$, which belong to case $4 \mathrm{~b}$. These runs have complex waveforms at the beginning, which causes the $f_{\mathrm{p}}$ to become smaller than that of the Helmholtz resonance. Excluding these 2 outliers, we fit the data to a power law relation $\beta \propto f_{\mathrm{p}}^{m}$ and obtain an exponent $m=1.66 \pm 0.01(r=0.948)$. This $m$ is closer to the $m=2$ predicted by radiation damping (Eq. (13)) than to the $m=0.5$ for visco-thermal damping (Eq. (14)). We calculated $\beta_{\mathrm{r}}, \beta_{\mathrm{vt}}$ using $R_{\mathrm{a}}=2$ and $7 \mathrm{~mm}$ which we 

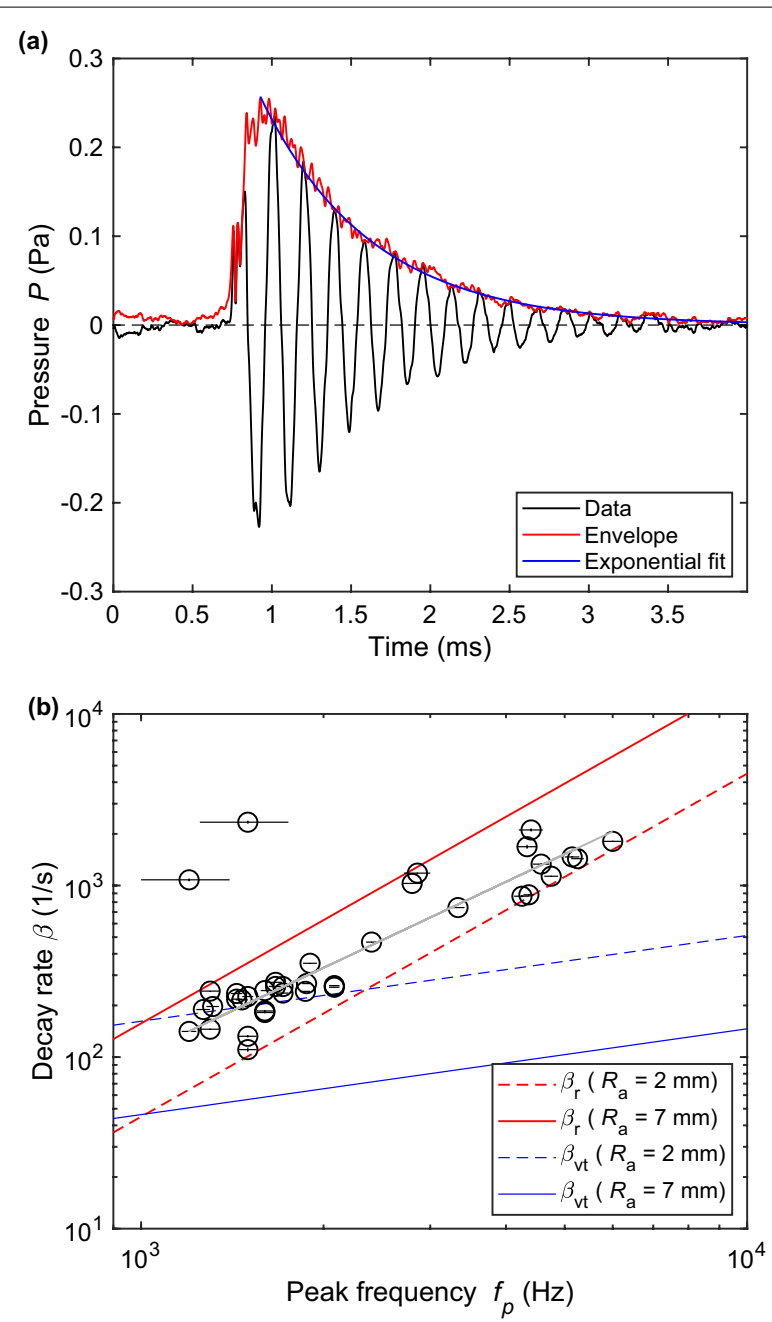

Fig. 13 Decay of Helmholtz resonance. a Waveform of the Helmholtz resonance excited by a $V=0.6 \mathrm{~cm}^{3}$ bubble bursting in a suspension of $\phi=0.4$. Red and blue lines indicate an envelope and its exponential fit, respectively from which we obtain $\beta=1.434 \pm 0.006$ $1 / \mathrm{ms}$. The waveform was detrended, and a highpass $(>800 \mathrm{~Hz})$, bandstop $(2000,4000 \mathrm{~Hz}$ ) filters were applied. b $\beta$ vs. peak frequency $f_{\mathrm{p}}$ (both with errors) for the case in which Helmholtz resonance was excited by a bubble bursting in a suspension of $\phi=0.4$. The gray line indicates a power law fit excluding 2 outliers (see text). Thick red and thin blue lines indicate the $\beta_{\mathrm{r}}$ and $\beta_{\mathrm{vt}}$ calculated from radiation (Eq. (13)) and visco-thermal (Eq. (14)) damping models, respectively, using the $R_{\mathrm{a}}$ values given in the legend

indicate in Fig. 13b. These $R_{\mathrm{a}}$ values were chosen so that $\beta_{\mathrm{r}}$ bounds the measured $\beta$, excluding the outliers, and are consistent with the measurements (Fig. 11a). We find that for $R_{\mathrm{a}}>3.5 \mathrm{~mm}$, radiation damping becomes the main mechanism $\left(\beta_{\mathrm{r}}>\beta_{\mathrm{vt}}\right)$. Visco-thermal damping is important $\left(\beta_{\mathrm{vt}}>\beta_{\mathrm{r}}\right)$ only during the initial stage of the aperture opening $\left(R_{\mathrm{a}}<2 \mathrm{~mm}\right)$ of a large $(V>2.7$ $\mathrm{cm}^{3}$ ) bubble (corresponding to $f_{\mathrm{p}}<2350 \mathrm{~Hz}$ ).

\section{Origin of high-frequency wave}

We do not fully understand the origin of the HF wave excited by a hemispherical cap bubble or the HF wave component excited by a rising bubble together with Helmholtz resonance. Airwaves that appear to be the same as an HF wave have been reported for a bubble bursting in water (Spiel 1992; Deane 2013) and in gel (Divoux et al. 2008). These works report that such a wave is excited when the subsurface bubble volume is small or when a longer time has elapsed after surfacing, which is similar to our result in which the HF wave is excited by a hemispherical cap bubble formed after bubble surfacing. Deane (2013) also reported that such wave is excited together with Helmholtz resonance, which is the same as our results for cases $4 \mathrm{~b}$ and 5 (Figs.6, 7, 8).

Our tentative interpretation is that it originates from the film vibration $f_{\mathrm{V}}$, which scales with the film thickness $h$ and volume $V$ as $f_{\mathrm{V}} \propto h^{-1 / 2} V^{-1 / 6}$ (Eq. (7)). In detail, we showed that the frequency of the HF wave and HF wave component correlate differently with $V$ (Fig. 12). Here we explain that this may have arisen from the effective $h$ depending differently on $V$. Images of the runs in which the HF wave component was excited show that when the bubble is small $\left(V \leq 4 \mathrm{~cm}^{3}\right)$, in all runs, the bubble bursts after a thin film patch forms near the bubble apex (e.g., Fig. 6). On the other hand, when the bubble is large $\left(V \geq 5 \mathrm{~cm}^{3}\right)$, in the $52 \%$ of the runs, the bubble bursts before a thin film patch forms (e.g., Fig. 7), indicating that its film is still thick. This observation suggests that the $h$ at burst increases with the $V$, which explains the negative correlation. On the other hand, a thin patch cannot be identified when the hemispherical cap bubble bursts (e.g., Fig. 5). We thus infer from a poor correlation with $V$, that the $h$ depend little on $V$. Direct measurement of $h$ is needed however, to verify this.

The film vibration model is nevertheless simplified. We note that this model does not include an aperture, whereas in our experiments, the HF wave is excited after the aperture opens. When the aperture becomes too large, the bubble can no longer be compressed to excite vibration. In addition, power spectra (Fig. 10) show that HF waves commonly consist of more than one frequency component, suggesting that vibration of higher modes may also be excited. We also note that the $\mathrm{N}$-wave (Eq. (10)) may also be excited, which explains the frequency of $\mathcal{O}\left(10^{4}\right) \mathrm{Hz}$ as shown in Fig. 12. However, our waveform is not an ideal $\mathrm{N}$-shape, and the aperture growth velocity $v_{\mathrm{a}}$ is in the range of $v_{\mathrm{a}}=0.3-9 \mathrm{~m} / \mathrm{s}$, which is much slower than the sound velocity $c$. This indicates that bursting is not instantaneous as assumed for the $\mathrm{N}$-wave, and for 
this case, Eq. (10) becomes an upper bound (Kulkarny 1978).

\section{Condition for the excitation of Helmholtz resonance}

Our experiments indicate that Helmholtz resonance is excited only when a bubble of $0.5 \leq V \leq 9 \mathrm{~cm}^{3}$ rises and bursts in a suspension of $\phi=0.4$ (Fig. 4). Here we consider the origin of this condition.

\section{HF wave - Helmholtz resonance transition}

First we consider the main transition indicated by a broken curve in Fig. 4b above which Helmholtz resonance is excited. Moitra et al. (2018) showed that when a dense suspension $(\phi=0.55)$ is elongated faster than the critical strain rate $\dot{\gamma}$, it fails brittly, whereas no fracturing occurs in a particle-free oil. Our experiments similarly show that when $V \geq 0.5 \mathrm{~cm}^{3}$ bubble rises in a $\phi=0.4$ suspension, the bubble bursts while it is rising when its film is still thick by being stretched faster. A close similarity indicates that the bubble film failed brittly above the critical $\dot{\gamma}$.

We show further evidence which indicate that the britthe failure does not occur in a particle-free oil. Previous works (Debrégeas et al. 1998; Nguyen et al. 2013) have shown that a bubble rising in high viscosity $(\eta=10-1000$ Pas) Newtonian silicone oil forms a hemispherical cap at the surface and bursts thereafter. This indicates that the bubble film does not fail brittly, even when the viscosity of the oil is high. To confirm, we conducted the same experiments using high viscosity ( $\eta=3.2,10.8$ Pas) silicone oils, which are liquid-like (Additional file 7: Fig. S5) and Newtonian, and the results are shown in Additional file 7: Fig. S10. Here a viscosity of $\eta=3.2$ Pas is the same as that for a $\phi=0.4$ suspension, which we estimate next. The experiments indeed showed that the bubble bursts after it forms a hemispherical cap and excites an HF wave.

Here we non-dimensionalize $\dot{\gamma}$ and define the Deborah number $\operatorname{De}(\dot{\gamma})$ for a shear-thinning Maxwell fluid,

$$
D e(\dot{\gamma})=\frac{t_{\mathrm{r}}}{t_{\mathrm{d}}}=\frac{\eta(\dot{\gamma}) \dot{\gamma}}{G_{\infty}}=\frac{\tau}{G_{\infty}} .
$$

$\operatorname{De}(\dot{\gamma})$ compares the relaxation time $t_{\mathrm{r}}=\eta(\dot{\gamma}) / G_{\infty}$, to the deformation time $t_{\mathrm{d}}=1 / \dot{\gamma}$, where $\dot{\gamma}$ is the strain rate of deformation. Here $\eta(\dot{\gamma})$ is the shear-thinning viscosity and $G_{\infty}$ is the infinite-frequency shear modulus, representing the viscous and elastic component, respectively and $\tau$ is the shear stress. For $D e \ll D e_{\mathrm{c}}$, the fluid deforms viscously; conversely for $D e \gg D e_{\mathrm{c}}$, it deforms elastically and fails brittly. Our solid-like suspension is elastic under small $\tau$ (Fig.1d), but fluidizes under the large $\tau$ (Fig. 1c) which is also apparent as shear-thinning $\eta(\dot{\gamma})$ (Additional file 7: Fig. S3). Together with the creep curves (Fig. 1e), this indicates that shortly after the stress is applied, its rheology can be approximated as a shear-thinning Maxwell fluid, from which we consider that Eq. (18) is applicable. Such rate-dependent $D e(\dot{\gamma})$ (and $t_{\mathrm{r}}$ ) has been used for shear-thinning silicic magmatic suspension (Cordonnier et al. 2012) and polymer (Arigo and McKinley 1998).

$D e$ has been used as a criterion for the viscous - britthe transition of magma and its analogues (see Wadsworth et al. (2017) for a review). Previous works have shown $D e_{\mathrm{c}} \sim 10^{-2}$ for silicate melts and syrup, and that $D e_{\mathrm{c}}$ criterion is applicable to a wide range covering at least $t_{\mathrm{r}}=\mathcal{O}\left(10^{-4}-10^{1}\right) \mathrm{s}$. Importantly, Cordonnier et al. (2012) showed that $D e_{\mathrm{c}}$ of magmatic suspension $(\phi=0.15-0.65)$ decreases with $\phi$ such that $D e_{\mathrm{c}}=\mathcal{O}\left(10^{-3}\right)$. Dingwell (1996) proposed a regime diagram mapping the flow - fragmentation transition in the $t_{\mathrm{d}}-t_{\mathrm{r}}$ parameter space. Our regime diagram (Fig. 4b) resembles his, which motivated us to evaluate $D e_{\mathrm{c}}$ at the transition.

Our method used to calculate $D e(\dot{\gamma})$ closely follows that of Cordonnier et al. (2012) who measured the $\tau$ (Eq. (18)) needed to deform a magmatic suspension under a controlled $\dot{\gamma}$, and used $G_{\infty}$ measured separately under a small strain $\gamma$ and high frequency $f$. Similarly we calculate $\tau=\eta(\dot{\gamma}) \dot{\gamma}$ for bubble film stretching and use $G^{\prime}$ measured under small amplitude oscillatory shear for $G_{\infty}$. High-speed images of the experiments in which Helmholtz resonance is excited show that before bursting, the bubble forms a hemisphere (height $R_{\mathrm{h}}$, surface area $S \sim 2 \pi R_{\mathrm{h}}^{2}$ ) above the surface, and its film is stretched as it rises. The areal strain rate $\dot{\gamma}$ of the film immediately before bursting can be expressed as

$$
\dot{\gamma} \sim \frac{1}{S} \frac{d S}{d t} \simeq \frac{2}{R_{\mathrm{h}}} U,
$$

where $U$ is the ascent velocity $U \sim d R_{\mathrm{h}} / d t$. From the images of rising $V=0.5 \mathrm{~cm}^{3}$ bubble, we obtain $R_{\mathrm{h}} \simeq 5.7$ $\mathrm{mm}, U \simeq 0.011 \mathrm{~m} / \mathrm{s}$, and substituting these values into Eq. (19), we obtain $\dot{\gamma} \simeq 3.71 /$ s; from Additional file 7: Fig. S3, we estimate $\eta=3.2$ Pas. For $G_{\infty}$, we use $G^{\prime}=8210 \mathrm{~Pa}(\phi=0.4)$ measured under $\gamma=10^{-4}$ (in the linear region) and $f=100 \mathrm{~Hz}$ (Fig. 1d) which best approximates the high frequency. Substituting these values we obtain $t_{\mathrm{r}} \sim 4 \times 10^{-4} \mathrm{~s}$ and $D e_{\mathrm{c}}=1.4 \times 10^{-3}$. The $D e_{\mathrm{c}}=\mathcal{O}\left(10^{-3}\right)$ is the same as $D e_{\mathrm{c}}$ obtained for $\phi=0.4$ silicic magmatic suspension (Cordonnier et al. 2012). Estimates of the $D e(\dot{\gamma})$ for other $V$ are given in the Additional file 7: Table S3, which shows that the $D e(\dot{\gamma})$ increases with $V$. This is consistent with the estimate $D e(\dot{\gamma}) \sim p / G_{\infty} \propto V^{1 / 3}$ derived from $\tau \sim p(p$ : bubble buoyancy). 
Next, we consider the transition between $\phi=0.3$ and $\phi=0.4$ suspension. $\phi=0.3$ suspension is also solid-like and non-Newtonian albeit weaker compared to those of $\phi=0.4$ suspension. We interpret this transition as a consequence of $D e_{\mathrm{c}}$ decreasing with $\phi$. From $\operatorname{De}(\dot{\gamma}) \propto V^{1 / 3} / G_{\infty}$ and since $G^{\prime}$ increases from $\phi=0.3$ to $\phi=0.4$ (Fig. 1d), it follows that for a given $V$, $D e(\dot{\gamma})$ decreases with $\phi$. Thus we inferred that the $D e_{\mathrm{c}}$ dropped with $\phi$ as shown by Cordonnier et al. (2012).

$D e(\dot{\gamma})$ defined in Eq. (18) assumes a simple viscoelasticity, but we consider it sufficient to explain the viscous - brittle transition we observe. Yield stress $\tau_{\mathrm{y}}$ need not be included in the criterion because it is small compared to the buoyancy pressure $p$ and capillary stress which we show below. As we showed in Fig. 1f, $\tau_{\mathrm{y}} \sim 4$ $\mathrm{Pa}$ of the $\phi=0.4$ suspension is more than 1 order of magnitude smaller than the $p \simeq 71 \mathrm{~Pa}\left(V_{\mathrm{c}}=0.5 \mathrm{~cm}^{3}\right)$ that drives bubble ascent and film stretching. $\tau_{\mathrm{y}}$ is also 2 - 3 orders of magnitude smaller than the capillary stress $2 \sigma / h \sim \mathcal{O}\left(10^{2}-10^{3}\right) \mathrm{Pa}$ (Debrégeas et al. 1998) that drives film retraction during aperture growth.

Nevertheless we emphasize that the universality of the value of $D e_{\mathrm{c}}$, its dependence on $\phi$, the liquid rheology, and the deformation style, are poorly understood. We also do not well understand the condition under which $D e$ is sufficient to explain the viscous - brittle transition noting that other criteria have been proposed (see Gonnermann and Manga (2013) for a review). We also note that the $t_{\mathrm{r}}$ of silicic magmatic suspension measured by Cordonnier et al. (2012) is 3 - 6 orders of magnitude larger than the $t_{\mathrm{r}}$ of our suspensions. Thus more suspension rheology measurements which determine the failure condition and its parameter dependence, covering a broad range of $t_{\mathrm{r}}$ are needed.

We estimate that a similar transition will occur if the same experiments which we did were conducted using other fluids (e.g., gel, syrup) which fail brittly above a critical $\dot{\gamma}$, provided that $D e$ is close to $D e_{\mathrm{c}}$ and the surface tension is small (a large Eo: Eq. (4)). Brittle failure is not required to excite Helmholtz resonance when Eo is small, because the static bubble protrudes less above the surface. This is the situation for a small bubble $(V \leq 0.14$ $\mathrm{cm}^{3}$ ) bursting in water (Spiel 1992; Deane 2013; Poujol et al. 2021). The $E o$ of bubbles in water used in previous works are in the range of $E o<6$, which is smaller than the $E o=\mathcal{O}\left(10^{1}-10^{2}\right)$ of bubbles used in our experiments. Since the maximum aperture radius $R_{\mathrm{a}}$ is less than the bubble surface radius $R_{\mathrm{b}}$, for the same $V$, the maximum $R_{\mathrm{a}}$ becomes smaller for a bubble in water. This seems to be geometrically favorable for the excitation of Helmholtz resonance.

\section{Helmholtz resonance - Air flow transition}

Next we consider the upper limit $V_{\mathrm{c}}=10 \mathrm{~cm}^{3}$, above which Helmholtz resonance is no longer excited and is dominated by air flow (case 6b). Here we emphasize that the air flow will always exist, but the pressure signal arising from air flow becomes dominant only for these large bubbles. For these large bubbles, the period of Helmholtz resonance becomes longer $(\sim 1 \mathrm{~ms})$, and the aperture grows faster (Fig. 11) than in the case of smaller bubbles. Thus $R_{\mathrm{a}}$ is no longer small relative to the bubble size, which is assumed for Helmholtz resonance and this may have suppressed its excitation.

\section{Simultaneous excitation of air column resonance}

Now we study the situation where a bubble bursts in a suspension whose level is further below the rim, such that air column resonance is simultaneously excited in addition to Helmholtz resonance.

\section{Frequency and decay rate of air column resonance}

The air column resonance frequency $f_{\mathrm{A}}$ in an openclosed pipe (length $L$ ) can be expressed by extending the formula in Kinsler et al. (2000) as

$$
f_{\mathrm{A}}=\frac{(2 n+1) c}{4(L+\delta L)}
$$

where $n=0$ indicates the fundamental mode, $n=1$ indicates the third-order mode and so on, $c$ is the sound velocity, and $\delta L$ is the open-end correction. For our square tank (half width $a=1.5 \mathrm{~cm}$ ) surrounded by a square flange (half width $2.5 \mathrm{~cm}$ ), $\delta L=0.802 a$ (Dalmont et al. 2001).

The decay rate $\beta_{\mathrm{a}}$ of an air column resonance $(n=0)$ due to radiation damping is expressed as a function of $L$ as

$$
\beta_{\mathrm{a}}=\frac{\pi f_{\mathrm{A}}}{Q(L)}=\frac{\pi^{2}}{16} \frac{a^{2} c}{L(L+\delta L)^{2}},
$$

where we used the expression for quality factor $Q(L)$ given in Johnson et al. (2018a). Both $f_{\mathrm{A}}$ and $\beta_{\mathrm{a}}$ have been used to constrain $L$ at volcanoes and to monitor their changes over time (Johnson et al. 2018b).

When both Helmholtz and air column resonances are simultaneously excited, their waveforms having different frequencies and decay rates, will be superimposed. The condition for the air column resonance to continue longer than the Helmholtz resonance can be expressed as

$$
\beta_{\mathrm{a}}<\beta_{\mathrm{r}}+\beta_{\mathrm{vt}},
$$



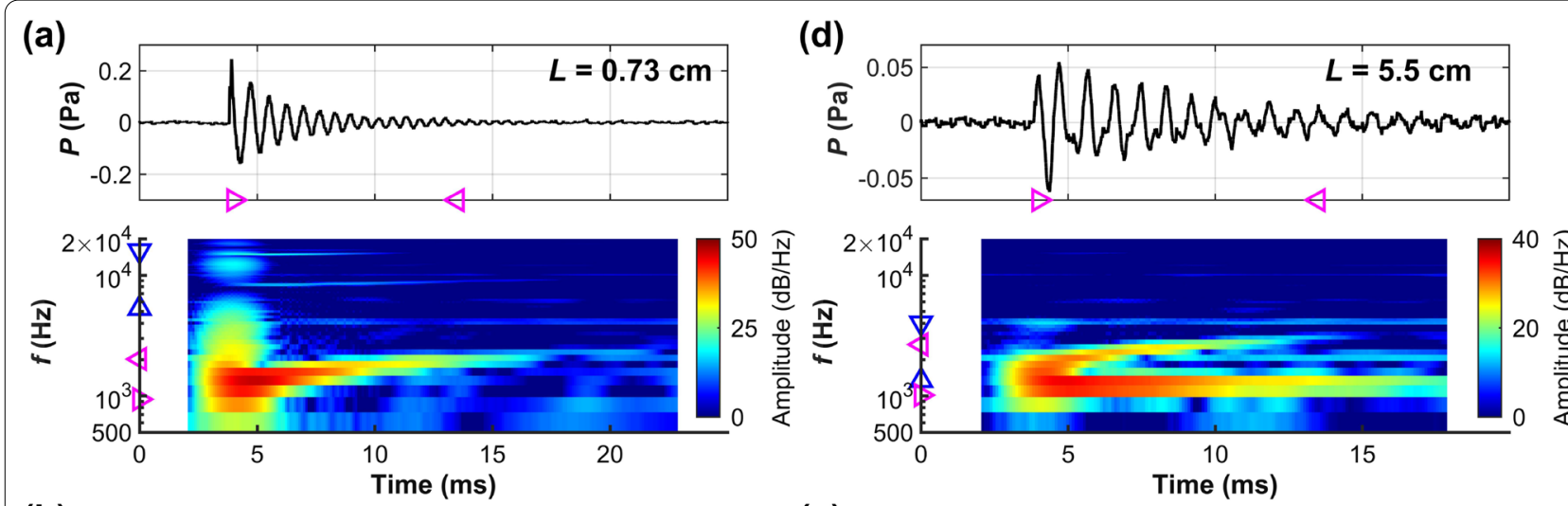

(b)

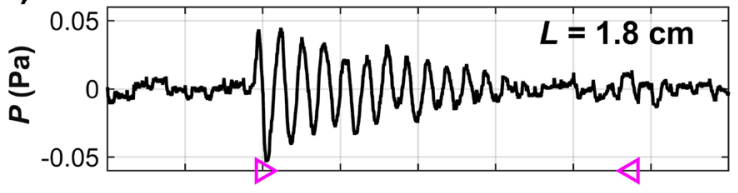

(e)
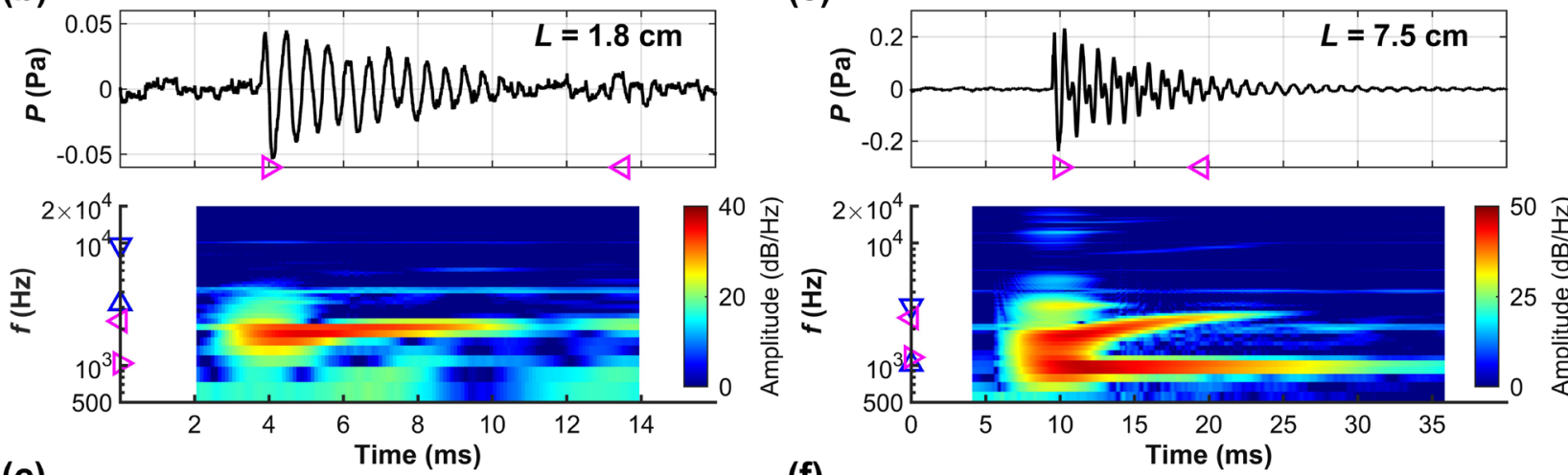

(c)

(f)
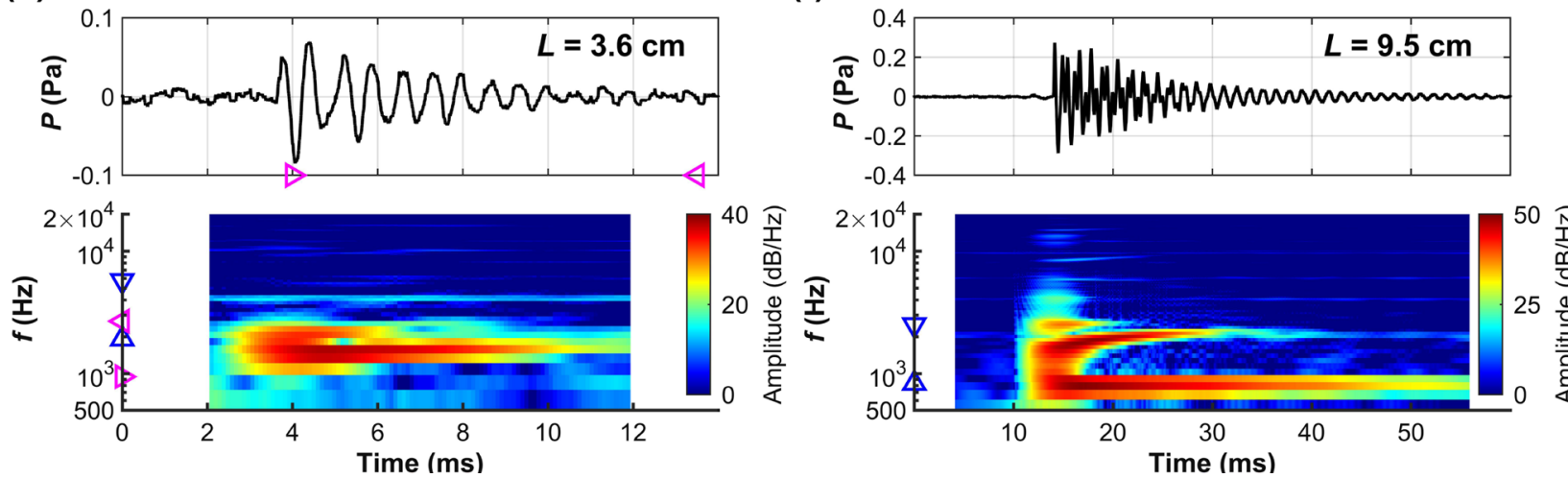

Fig. 14 Waveforms and spectrograms (re $20 \mu \mathrm{Pa}^{2}$ ) of airwaves excited at varying levels. $V=5 \mathrm{~cm}^{3}$ bubble bursts in a suspension of $\phi=0.4$. Air column lengths $L(\mathrm{~cm})$ are annotated (see Additional file 6: Movie 6 and the Graphical Abstract for the images of (e)). Waveforms are detrended and highpass filtered at $500 \mathrm{~Hz}$. Running windows and overlaps are (a-d) $4.096 \mathrm{~ms}$ and $4.000 \mathrm{~ms}$, (e-f) $8.192 \mathrm{~ms}$ and $8.100 \mathrm{~ms}$, respectively. Blue $\Delta$ and $\nabla$ indicate the $f_{\mathrm{A}}\left(n=0,1\right.$, respectively) calculated from Eq. (20). Pink $\triangleright$ and $\triangleleft$ indicate the $f_{\mathrm{H}}$ calculated from Eq. (11) using aperture radii $R_{\mathrm{a}}$ measured at the times indicated on the time axis. In $(\mathbf{f}), f_{H}$ is not calculated because the timing of the aperture opening could not be determined from the images

where the right-hand side indicates the decay of Helmholtz resonance resulting from radiation $\left(\beta_{\mathrm{r}}\right.$, Eq. (13)) and visco-thermal $\left(\beta_{\mathrm{vt}}\right.$, Eq. (14)) dampings. This inequality can be used to estimate the critical air column length $L_{\mathrm{c}}$ above which the air column resonance continues longer than the Helmholtz resonance.
Air column length dependence of waveform, spectrum and decay rate

Figure 14 shows how the waveforms of the excited airwaves and their spectrograms change as $L$ increases from $L=0.73 \mathrm{~cm}$ to $L=9.5 \mathrm{~cm}$, as the suspension level is lowered (Fig. 2). Here a large bubble (slug) with a fixed 


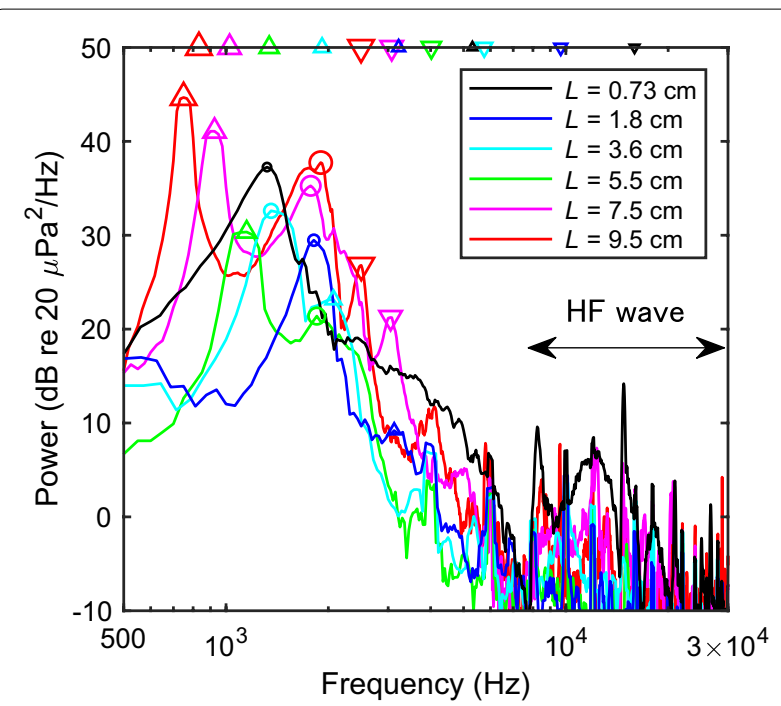

Fig. 15 Power spectra of the waveforms shown in Fig. 14. Bandstop filters were further applied at 2000 and $4000 \mathrm{~Hz} . \Delta, \nabla$, and $\bigcirc$, indicate the peaks which we identified as air column resonances $(n=0,1)$ and Helmholtz resonance, respectively. $\Delta$ and $\nabla$ plotted on the upper frequency axis indicate the $f_{A}$ calculated from Eq. (20) using $n=0,1$ and their sizes correspond to $L$

$V\left(=5 \mathrm{~cm}^{3}\right)$ rises in a suspension of $\phi=0.4$ and excites Helmholtz resonance (case 5 in regime III).

Figure 14a shows the result for $L=0.73 \mathrm{~cm}$. For this case, the waveform consists of a signal arising from Helmholtz resonance, and the spectrogram shows gliding toward a higher frequency as a result of aperture growth. The HF wave component $(>10000 \mathrm{~Hz})$ is also excited upon bursting, the same as in Fig. 6c. From the images, we measured the aperture radius $R_{\mathrm{a}}$ at the beginning and end of the resonance, and their times are marked on the time axis of the waveform. Using these $R_{\mathrm{a}}$, we calculated the $f_{\mathrm{H}}$ values (Eq. (11)), which are plotted on the frequency axis of the spectrogram. The calculated $f_{\mathrm{H}}$ explains the measured peak frequency and gliding. We also calculated the air column resonance frequencies $f_{\mathrm{A}}$ (Eq. (20)), which are indicated on the frequency axis of the spectrogram. However, the signal indicating air column resonance could not be identified on the spectrogram.

Figure 14c-f shows that as $L$ increases, the waveform becomes complex, and another frequency component appears on the spectrogram. Unlike Helmholtz resonance, the frequency of this component does not change with time and, importantly, decreases with $L$. Since the frequencies of this component agree fairly well with the calculated $f_{\mathrm{A}}$, we interpret that they correspond to the air column resonance. We find that $n=0$ and $n=1$ modes are excited at $L \geq 1.8 \mathrm{~cm}$ and $L \geq 7.5 \mathrm{~cm}$, respectively.

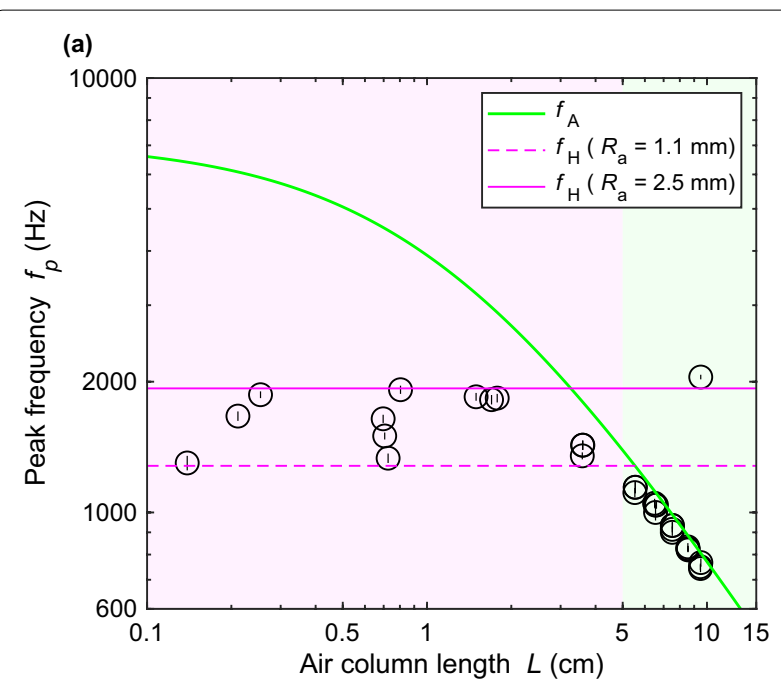

(b)

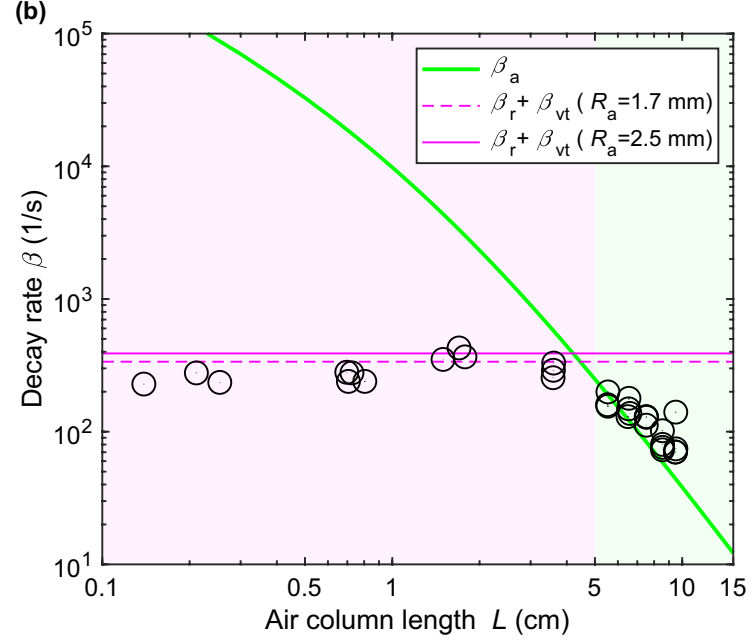

Fig. 16 Peak frequency $f_{p}$, decay rate $\beta$ (both with errors) vs. air column length $L$. a $f_{\mathrm{p}} v \mathrm{v}$. $L$ of airwaves excited by $\mathrm{a} V=5 \mathrm{~cm}^{3}$ bubble bursting in a suspension of $\phi=0.4$. A green curve indicates $f_{\mathrm{A}}$ (Eq. (20)). Pink lines indicate $f_{H}(E q .(11))$ calculated using $R_{a}$ values given in the legend. $\mathbf{b} \beta$ vs. L; otherwise the same as (a). A green curve indicates the decay rate $\beta_{\mathrm{a}}$ (Eq. (21)). Pink lines indicate $\beta_{\mathrm{r}}+\beta_{\mathrm{vt}}$ (Eqs. (13), (14)) calculated using $R_{a}$ values given in the legend. $R_{a}$ is chosen to calculate the minimum and maximum values of $\beta_{\mathrm{r}}+\beta_{\mathrm{vt}}$ in the range of $R_{\mathrm{a}}=1.1-2.5 \mathrm{~mm}$ used in (a)

For $L \geq 7.5 \mathrm{~cm}$, the air column resonance continues for a long time, $\sim 3-50 \mathrm{~ms}$.

Figure 15 compares the corresponding power spectra. The figure shows that since $V$ is fixed, the peak frequencies $f_{\mathrm{p}}$ of Helmholtz resonance $(\bigcirc)$ are also fixed in the $1000-2000 \mathrm{~Hz}$ band. On the other hand, $f_{\mathrm{p}}$ corresponding to the air column resonance decrease with $L$ and agree fairly well with the calculated $f_{\mathrm{A}}$. The spectra show that for $L \leq 3.6 \mathrm{~cm}$, the primary $f_{\mathrm{p}}$ corresponds to $f_{\mathrm{H}}(\bigcirc)$, whereas for $L \geq 5.5 \mathrm{~cm}$, it corresponds to $f_{\mathrm{A}}$ $(\triangle)$, indicating that the power of air column resonance 
Table 2 Dimensionless numbers for a rising bubble : the Eötvös Eo $=\Delta \rho g d_{\text {be }}^{2} / \sigma$ (bubble size, Eq. (4)), the Morton $M o=g \eta^{4} / \rho \sigma^{3}$ (fluid property), and the Reynolds $R e=\rho U d_{\mathrm{be}} / \eta$ (bubble velocity) numbers (see Tables 3, 4 for notations). For magma, $\eta=230-1.7 \times 10^{4}$ Pas (Additional file 7: Material), $d_{\mathrm{be}}=1-10 \mathrm{~m}$ are assumed and the Re is calculated from the Eo, Mo using an empirical function (Clift et al. 1978)

\begin{tabular}{lllll}
\hline Case & Eo & Mo & Re & flow type \\
\hline Expt. $(\phi=0.4)$ & $20-520$ & $3 \times 10^{3}-1 \times 10^{8}$ & $8 \times 10^{-4}-5$ & viscous \\
Expt. (all) & $20-520$ & $0.1-1 \times 10^{8}$ & $8 \times 10^{-4}-1 \times 10^{2}$ & viscous - transitional \\
Basaltic magma & $7 \times 10^{4}-7 \times 10^{6}$ & $2 \times 10^{8}-7 \times 10^{15}$ & $0.02-800$ & viscous - inertial \\
\hline
\end{tabular}

becomes larger. The power of the air column resonance increases with $L$, whereas that of the Helmholtz resonance does not show $L$ dependence.

Figure 16 shows how the peak frequency $f_{\mathrm{p}}$ and decay rate $\beta$ depend on $L$. Here we used all available data for a $V=5 \mathrm{~cm}^{3}$ bubble bursting in a suspension of $\phi=0.4$ and excited Helmholtz resonance (case 5). We determined $f_{\mathrm{p}}$ and $\beta$, the same as in Fig. 13(b). We also calculated $f_{\mathrm{A}}, \beta_{\mathrm{a}}$ for air column resonance and $f_{\mathrm{H}}, \beta_{\mathrm{r}}+\beta_{\mathrm{vt}}$ for Helmholtz resonance, which are shown in the figure. Based on our measurements, we define 2 regimes separated at $L=5 \mathrm{~cm}$ : a Helmholtz resonance dominant regime $(L<5 \mathrm{~cm})$ and an air column resonance dominant regime $(L>5 \mathrm{~cm})$, which are indicated as different background colors in the figure.

In the Helmholtz resonance dominant regime, $f_{\mathrm{p}}$ and $\beta$ do not depend on $L$. $f_{\mathrm{p}}=1300-1917 \mathrm{~Hz}$ can be explained by $f_{\mathrm{H}}$ with $R_{\mathrm{a}}=1.1-2.5 \mathrm{~mm}$, as shown in Fig. 16a. To check that this range of $R_{\mathrm{a}}$ is reasonable, we measured the $R_{\mathrm{a}}$ of 10 runs (case $5, V=5 \mathrm{~cm}^{3}$ ) and found that for all of these runs, $R_{\mathrm{a}}$ grows and exceeds $2.5 \mathrm{~mm}$ within $0.5-9.5 \mathrm{~ms}$ after bursting (Fig. 11a). This time scale is comparable to the mean decay time $1 / \beta \sim 3.5 \mathrm{~ms}$ obtained from $L<5 \mathrm{~cm}$ data, indicating that the above values of $R_{\mathrm{a}}$ are reasonable. Measured $\beta=293 \pm 59(1 / \mathrm{s})$ agrees fairly well with $\beta_{\mathrm{r}}+\beta_{\mathrm{vt}}$ calculated from the aperture radius of $R_{\mathrm{a}}=1.7,2.5 \mathrm{~mm}$ as indicated in Fig. 16b (see legend for the choice of these $R_{\text {a values). }}$

In the air column resonance dominant regime, $f_{\mathrm{p}}$ and $\beta$ decrease with $L$, and the measured $f_{\mathrm{p}}$ and $\beta$ agree fairly well with the calculated $f_{\mathrm{A}}$ and $\beta_{\mathrm{a}}$. Figure $16 \mathrm{~b}$ shows that $\beta_{\mathrm{r}}+\beta_{\mathrm{vt}}$ intersects with $\beta_{\mathrm{a}}$ at $L \sim 5 \mathrm{~cm}$. This corresponds to the condition given in Eq. (22) and thus explains critical $L$ well. We notice, however, that in Fig. 16a, there is an outlier at $L=9.5 \mathrm{~cm}, f_{\mathrm{p}}=2050 \mathrm{~Hz}(\mathrm{PSD}=37.78 \mathrm{~dB} /$ $\mathrm{Hz}$ ), which is close to $f_{\mathrm{H}}$ rather than to $f_{\mathrm{A}}$. The power spectrum of this data has a secondary peak at $750 \mathrm{~Hz}$ (PSD $=35.94 \mathrm{~dB} / \mathrm{Hz}$ ), which is close to the estimated $f_{\mathrm{A}}=830 \mathrm{~Hz}$. Thus, for this case, although $L>5 \mathrm{~cm}$, the
PSD of the Helmholtz resonance was larger than that of the air column resonance.

To summarize, our experiments showed that when Helmholtz resonance is excited at a lowered suspension level, air column resonance is excited simultaneously. When $L$ exceeds critical, the air column resonance determines the decay rate, corresponding to the condition $\beta_{\mathrm{a}}<\beta_{\mathrm{r}}+\beta_{\mathrm{vt}}$.

\section{Implications for basaltic volcanic eruptions}

First we explain the two effects which were not modeled in our experiments. One is the inertial effect. The Re (Table 2) indicates that the experiments model a bubble rising viscously in basaltic magma. Another is the bubble overpressure $\Delta P$ which can arise by ascending from depth, in addition to that which originates from surface tension. It is difficult to accurately estimate $\Delta P$, but a static model (James et al. 2009; Del Bello et al. 2012) can be used to obtain the limiting criterion for $\Delta P$ to arise. A bubble rising in a wide conduit expands in accord to the decrease in the static pressure, and is stable (Leighton 1994). However when a slug rises in a narrow conduit, the fluid above the slug partly drains as a falling film. As a result, a slug longer than critical becomes unstable and overpressurized. A maximum slug length $l_{\text {lim }}$ (at $1 \mathrm{~atm}$ ) which can rise to the surface without $\Delta P$ can be defined. For basaltic magma in a conduit, we estimate $l_{\text {lim }} \sim 10 \mathrm{~m}$ (Additional file 7: Material). A static model gives a lower limit estimate of $\Delta P$.

Next we explain the applicability and similarity of the experiments to volcanoes. It follows that our experiments approximate a $d_{\text {be }} \sim \mathcal{O}(0.1-1) \mathrm{m}$ bubble rising in a basaltic magma, such that Eo $\gg 1$ (Table 2), $R e \leq 10^{2}$ (laminar flow), and $\Delta P \ll P_{\mathrm{a}} \sim 10^{5} \mathrm{~Pa}$. The $D e_{\mathrm{c}}=1.4 \times 10^{-3}$ (Eq. (18)) which we obtained, is comparable to the $D e_{\mathrm{c}}$ obtained in the previous works. Thus it may be used to estimate the stress $\tau \sim D e_{\mathrm{c}} G_{\infty}$ needed for a magmatic bubble film to fail brittly, or the modulus $G_{\infty} \sim \tau / D e_{c}$ of the magma, when the $G_{\infty}$ or the $\tau$ is 
known. We remark that the experiments and volcano share a geometrical similarity. In the experiments, the $d_{\text {be }}$ scaled to the conduit width $W$ was $d_{\text {be }} / W=0.19-0.95$, which includes a slug $\left(d_{\mathrm{be}} / W>0.6\right)$, a geometry common at volcanoes. The experiments in which a slug bursts at different levels $L$ below the conduit rim were conducted for $L / W=0.24-3.2$, which covers a wide range including the case for Aso $(L / W \sim 2.4$ ) (Yokoo et al. 2019).

Now we describe the implications. Our regime diagram (Fig.4b) showed that the HF wave is excited in suspensions of all $\phi$ which we used and for the widest range of bubble volume $V$, where we emphasize that the HF wave component is excited together with the Helmholtz resonance (e.g., Figs.6d, 8b). This suggests that the HF wave, excited at burst, may be also common at volcanoes. On the other hand, the Helmholtz resonance is excited only when a bubble bursts while it is rising, which occurs in a solid - like $(\phi=0.4)$ suspension and for a $V$ above critical. This suggests that if the Helmholtz resonance could be detected at volcanoes, it may be used to track the change of magma rheology or bubble $V$. Here we may estimate the aperture radius $R_{\mathrm{a}}$, by combining the measured frequency $f_{\mathrm{p}}$ and decay rate $\beta$ (Fig. 13b), and then estimate the $V$, an analyses similar to that by Vergniolle and Caplan-Aucherbach (2004); Montalto et al. (2010). It is to be noted that there has been an observation at a supraglacial pond, suggestive of Helmholtz resonance in a bubble (Podolskiy 2020). We showed that an air flow vented from a deflating bubble can be detected (Fig. 6e, Additional file 7: Fig. S7) and that we may estimate its lower limit velocity $v_{\text {af }}$ from the time lag of the air flow. This analyses may complement the ballistic method used to estimate the $v_{\mathrm{af}}$ (Tsunematsu et al. 2019). We showed that when the fluid level is low, an air column resonance including its higher modes (frequency $f_{\mathrm{A}}$ ) is triggered by bubble bursting, which itself excites resonant frequencies that differs from $f_{\mathrm{A}}$ (Fig. 15). This suggests that the air column resonance at volcanoes (Johnson et al. 2018b) may be common.

Here we analyze the infrasound observed at Aso and compare with the experiments, bearing in mind that the experiments approximate a bubble with a small overpressure $\left(\Delta P \ll P_{\mathrm{a}} \sim 10^{5} \mathrm{~Pa}\right)$. An estimate of $\Delta P$ can be obtained from energy conservation (Wilson 1980). Using the maximum gas flow velocity at Aso (Tsunematsu et al. 2019), we estimate $\Delta P \sim 7 \times 10^{3} \mathrm{~Pa}$ (Additional file 7 : Materials), an upper limit using this method. Although $\Delta P<P_{\mathrm{a}}$, this $\Delta P$ is not negligible compared to the buoyancy pressure $p \sim \mathcal{O}\left(10^{4}\right)$ Pa of a meter-sized bubble. Thus for a bubble rising at Aso, the film stretching will be enhanced by the $\Delta P$. This differs from the experiments where the the film is stretched solely by the $p$.

Figure $17 \mathrm{a}, \mathrm{b}$ is an example of an eruptive event observed at Aso (Ishii et al. 2019). The spectrum (black line in Fig. 17c) shows a primary peak at $f_{\mathrm{p}}=0.5 \mathrm{~Hz}$, which has been interpreted as $f_{\mathrm{A}}$ with $L \sim 120 \mathrm{~m}$ (Yokoo et al. 2019). It appears similar to Fig. $15(L=5.5 \mathrm{~cm}$; $L / W=1.8$ ) whose primary peak is also $f_{\mathrm{A}}$. We analyzed a total of 10 events ( $30 \mathrm{~s}$ each) covering a total time span of 44 minutes and stacked the power spectra, shown as a red line in Fig. 17c. The stacked spectrum shows that the 0.5 and $2.2 \mathrm{~Hz}$ peaks are well-defined. However, the peaks within the $3.5-16.5 \mathrm{~Hz}$ band vary among events. Figure $17 \mathrm{c}$ also shows the background spectrum during the low activity period between the events. In addition to the incessant $f_{\mathrm{A}} \sim 0.5 \mathrm{~Hz}$ peak (Yokoo et al. 2019), the $\sim 2.2 \mathrm{~Hz}$ peak also exists. Comparing the spectra, we infer that the $3.5-16.5 \mathrm{~Hz}$ band (red arrow) originates from the eruptive event. The $\sim 2.2 \mathrm{~Hz}$ peak deviates from the predicted third-order $f_{\mathrm{A}} \sim 1.5 \mathrm{~Hz}$ of the air column resonance. Unlike the experiments, the Helmholtz resonance in a bubble is unsuited as a mechanism. We speculate that it may be a resonance in a separate cavity.

Since the $3.5-16.5 \mathrm{~Hz}$ band appears similar to the HF wave excited at burst, we estimate the bubble radius $R_{\mathrm{b}}$ which excites this band, assuming $f_{\mathrm{V}}$ and $f_{\mathrm{N}}$. From Eq. (7), we find that a $R_{\mathrm{b}} \sim 3 \mathrm{~m}$ bubble excites $f_{\mathrm{V}} \sim 10 \mathrm{~Hz}$, where we assumed a film thickness of $h \sim 30 \mathrm{~mm}$ from the scoriae size (Namiki et al. 2018) (see Additional file 7: Materials for other parameters). This bubble is spherical because it is smaller than the conduit radius $W / 2 \sim 25$ $\mathrm{m}$ (Yokoo et al. 2019). The observed frequency range may be explained by the variation of $R_{\mathrm{b}}$ and $h$ (Fig. 12). From Eq. (10), we find that a $R_{\mathrm{b}} \sim 20 \mathrm{~m}$ bubble excites $f_{\mathrm{N}} \sim 16$ $\mathrm{Hz}$, where we assumed $c=650 \mathrm{~m} / \mathrm{s}$ for a hot gas with ash (Ishii et al. 2019). Thus both $f_{\mathrm{V}}, f_{\mathrm{N}}$ can become candidates to explain this band.

\section{Conclusions}

Experiments on bubble bursting and airwave excitation revealed 2 strikingly different regimes. When a bubble rises in a suspension of $\phi \leq 0.3$ or a small bubble $\left(V \leq 0.4 \mathrm{~cm}^{3}\right)$ rises in a suspension of $\phi=0.4$, it forms a hemispherical cap after surfacing and bursts thereafter, exciting an HF wave, which is likely to be excited by film vibration. In contrast when a large bubble $(V \geq 5$ $\mathrm{cm}^{3}$ ) rises in a suspension of $\phi=0.4$, it bursts when it partially protrudes above the surface, and excites Helmholtz resonance and/or vents a detectable air flow. An intermediate-sized bubble $\left(0.5 \leq V \leq 4 \mathrm{~cm}^{3}\right)$ rising in 


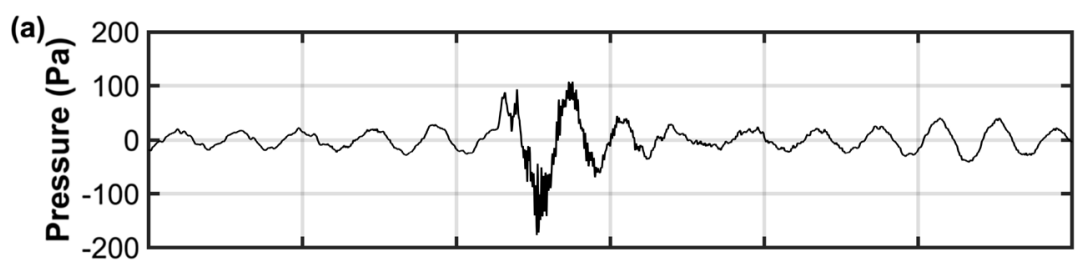

(b)

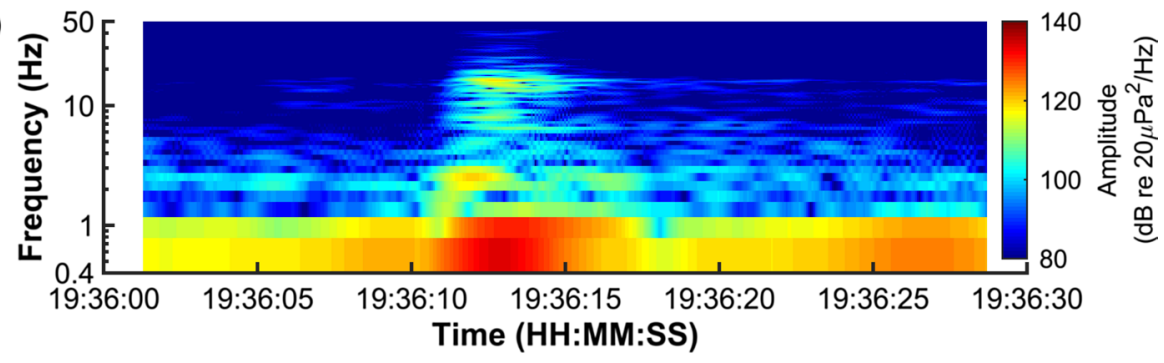

(c)

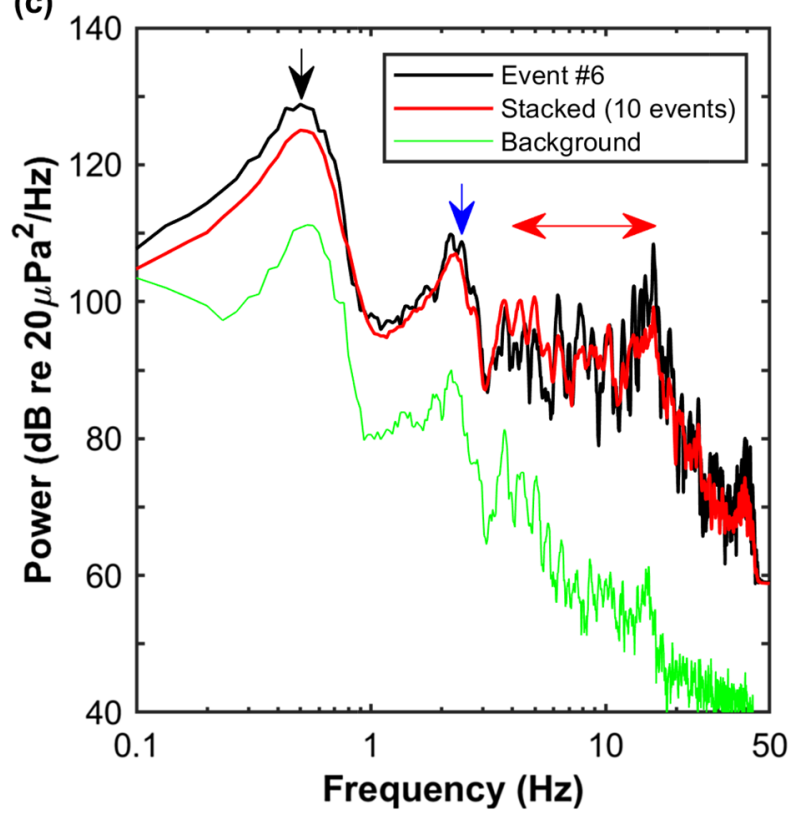

Fig. 17 Infrasound observed at the Aso volcano, Japan, on April 21, 2015 (JST) (Ishii et al. 2019). a An example of a waveform (detrended). b Spectrogram of (a) (time window $2.56 \mathrm{~s}$, overlap $2.50 \mathrm{~s}$ ). c Power spectrum (smoothed) of (a) (event\#6), stacked spectrum of 10 events during 19:07:10 - 19:51:00 (JST), background between the events (19:26:00 - 19:26:30). Arrows indicate the peaks at 0.5, $2.2 \mathrm{~Hz}$, and the $3.5-16.5 \mathrm{~Hz}$ band

a suspension of $\phi=0.4$, bursts with a mixture of above 2 styles. We interpreted that the regime transition corresponds to the viscous - brittle transition, and estimated the critical Deborah number as $D e_{\mathrm{c}}=1.4 \times 10^{-3}$, which is comparable to the $D e_{\mathrm{c}}$ at the viscous - brittle transition of a silicic magmatic suspension. When a bubble excites Helmholtz resonance in the conduit with a lowered suspension level, an air column resonance is excited simultaneously. The critical air column length $L$ above which the air column resonance continues longer than the 
Table 3 Notation

\begin{tabular}{|c|c|}
\hline$a$ & tank or conduit half width (m) \\
\hline c & sound velocity (m/s) \\
\hline d & diameter (m) \\
\hline$f$ & frequency $(\mathrm{Hz})$ \\
\hline G & modulus $(\mathrm{Pa})$ \\
\hline$g$ & gravitational acceleration $\left(\mathrm{m} / \mathrm{s}^{2}\right)$ \\
\hline K & consistency $\left(\operatorname{Pa} s^{\eta}\right)$ \\
\hline$h$ & film thickness (m) \\
\hline L & air column or cavity length (m) \\
\hline$\delta L$ & open-end correction (m) \\
\hline 1 & submerged bubble length (m) \\
\hline$l^{\prime}$ & effective neck length (m) \\
\hline$m$ & power law exponent $\left(\beta \propto f_{\mathrm{p}}^{m}\right)$ \\
\hline$n$ & flow index \\
\hline $\mathcal{O}$ & the order of \\
\hline$P$ & pressure $(\mathrm{Pa})$ \\
\hline$\Delta P$ & overpressure (Pa) \\
\hline$p$ & bubble buoyancy pressure $(\mathrm{Pa})$ \\
\hline R & radius $(m)$ \\
\hline$r$ & correlation coefficient \\
\hline$t$ & time $(s)$ \\
\hline U & ascent velocity $(\mathrm{m} / \mathrm{s})$ \\
\hline V & bubble volume $\left(\mathrm{m}^{3}\right)$ \\
\hline$v$ & velocity $(\mathrm{m} / \mathrm{s})$ \\
\hline w & tank or conduit width (m) \\
\hline$\beta$ & decay rate $(1 / \mathrm{s})$ \\
\hline$\dot{\gamma}$ & strain rate $(1 / \mathrm{s})$ \\
\hline$\gamma_{\mathrm{h}}$ & ratio of specific heats \\
\hline$\eta$ & viscosity (Pa s) \\
\hline$\rho$ & density $\left(\mathrm{kg} / \mathrm{m}^{3}\right)$ \\
\hline$\Delta \rho$ & density difference $\left(\mathrm{kg} / \mathrm{m}^{3}\right)$ \\
\hline$\sigma$ & surface tension coefficient $(\mathrm{N} / \mathrm{m})$ \\
\hline$\phi$ & volumetric packing fraction \\
\hline$\tau$ & shear stress $(\mathrm{Pa})$ \\
\hline
\end{tabular}

Helmholtz resonance is explained by the decay rate of the former becoming less than that of the latter. Our work provides an experimental evidence that a bursting bubble excites diverse airwaves, including the triggering of an air column resonance. The airwaves cover a frequency range of 3 orders of magnitude, suggesting that the same broadband excitation occurs at basaltic volcanoes. The HF wave is commonly excited, whereas the Helmholtz resonance is excited under a limited condition. This implies that if the Helmholtz resonance could be detected, it may be used to track the change of magma rheology or bubble $V$.

\section{Supplementary Information}

The online version contains supplementary material available at https://doi. org/10.1186/s40623-021-01472-7.
Table 4 Subscripts

\begin{tabular}{ll}
\hline A & air column resonance \\
af & air flow \\
a & aperture, air column, air \\
b & bubble \\
be & equivalent bubble \\
C & critical \\
d & deformation \\
H & Helmholtz resonance \\
i & inviscid \\
I & liquid \\
N & N-wave \\
P & particle, peak \\
r & relative, radiation, relaxation \\
V & film vibration \\
v & viscid \\
vt & visco-thermal \\
y & yield \\
\hline
\end{tabular}

Additional file 1: Movie 1. An example of a bursting bubble dominantly exciting an $\mathrm{HF}$ wave (case 3) shown in Fig. 5. A hemispherical cap bubble of a volume $V=0.6 \mathrm{~cm}^{3}$ bursts in a suspension of $\phi=0.3$ (air column length $L \sim 1 \mathrm{~mm}$ ) and the aperture grows rapidly. Replayed at $1 / 3000$ speed

Additional file 2: Movie 2. An example of a bursting bubble dominantly exciting Helmholtz resonance (case 5) shown in Fig. 6. A bubble of a volume $V=0.6 \mathrm{~cm}^{3}$ bursts while it is rising in a suspension of $\phi=0.4$ $(L<1 \mathrm{~mm})$. The aperture grows slowly compared to Movie 1. Replayed at 1/200 speed (1/1000 during slowdown motion).

Additional file 3: Movie 3. An example of a bursting bubble dominantly exciting Helmholtz resonance (case 5) shown in Fig. 7. A bubble of a volume $V=7 \mathrm{~cm}^{3}$ bursts while it is rising in a suspension of $\phi=0.4$ $(L \sim 7 \mathrm{~mm})$. The aperture grows slowly compared to Movie 1. Replayed at $1 / 500(1 / 100)$ speed at $t<15 \mathrm{~ms}(t>15 \mathrm{~ms})$.

Additional file 4: Movie 4. An example of a bursting bubble exciting Helmholtz resonance after a thin film bursts (case 4b) shown in Fig. 8. A bubble of a volume $V=6 \mathrm{~cm}^{3}$ bursts while it is rising in a suspension of $\phi=0.4(L \sim 6 \mathrm{~mm})$. A thin film forms at the top of the bubble, which bursts and the aperture grows rapidly compared to the cases shown in Movies $2-3$. Replayed at 1/2000 speed.

Additional file 5: Movie 5. An example of a bursting bubble dominantly exciting an air flow (case 6a) shown in Fig. 9. A bubble of a volume $V=12 \mathrm{~cm}^{3}$ bursts while it is rising in a suspension of $\phi=0.4$ $(L \sim 13 \mathrm{~mm}$ ). The aperture grows rapidly compared to Movies $2-3$. A pink arrow on the spectrogram indicates Helmholtz resonance. Replayed at $1 / 100$ speed.

Additional file 6: Movie 6. A bubble of a volume $V=5 \mathrm{~cm}^{3}$ bursts while it is rising in a suspension of $\phi=0.4$ with a lowered suspension level (air column length $L=7.5 \mathrm{~cm}$ ), shown in Fig. 14e and in the Graphical Abstract. The air column resonance $(n=0, n=1)$, the Helmholtz resonance, and an HF wave are simultaneously excited. Replayed at 1/400 speed.

Additional file 7. Additional materials, tables and figures. 


\section{Acknowledgements}

We thank Roberto Sulpizio and anonymous reviewers for their helpful comments which improved the manuscript, A. Namiki for discussions, A. Yokoo and K. Ishii for providing a preprint and infrasound data, and K. Tsunematsu for a video of eruption.

\section{Authors' contributions}

$\mathrm{KH}$ conducted the experiments and data analyses. IS contributed to devising the experimental method and modeling the results. Both authors contributed to interpreting the data, preparing and revising the manuscript. Both authors read and approved the final manuscript.

\section{Funding}

This work was supported by JSPS KAKENHI (JP15K13591, JP17H05417, JP18K03800) and Sasakawa Scientific Research Grant (2018-6020).

\section{Availability of data and materials}

The data used in this study are available from IS.

\section{Declarations}

\section{Competing interests}

The authors declare that they have no competing interests.

Received: 24 March 2021 Accepted: 24 June 2021

Published online: 13 July 2021

\section{References}

Arigo MT, McKinley GH (1998) An experimental investigation of negative wakes behind spheres settling in a shear-thinning viscoelastic fluid. Rheol Acta 37:307-327

Bonn D, Denn MM, Berthier L, Divoux T, Manneville S (2017) Yield stress materials in soft condensed matter. Rev Mod Phys. https://doi.org/10.1103/Rev/ ModPhys.89.035005

Clift R, Grace JR, Weber ME (1978) Bubbles, Drops, and Particles. Dover, New York Cordonnier B, Caricchi L, Pistone M, Castro J, Hess K-U, Gottschaller S, Manga M, Dingwell DB, Burlini L (2012) The viscous-brittle transition of crystal-bearing silicic melt : direct observation of magma rupture and healing. Geology 40:611-614

Culick FEC (1960) Comments on a ruptured soap film. J Appl Phys 31:1128-1129. https://doi.org/10.1063/1.1735765

Dalmont J-P, Nederveen CJ, Joly N (2001) Radiation impedance of tubes with different flanges : numerical and experimental investigations. J Sound Vibration 244:505-534

Deane GB (2013) Determining the bubble cap film thickness of bursting bubbles from their acoustic emissions. J Acoust Soc Am 133:EL69

Debrégeas G, de Gennes P-G, Brochard-Wyart F (1998) The life and death of "bare" viscous bubbles. Science 279:1704-1707

Deihl DT, Carlson FR (1968) "N waves" from bursting balloons. Am J Phys 36:441-444

Del Bello E, Llewellin EW, Taddeucci J, Scarlato P, Lane S (2012) An analytical model for gas overpressure in slug-driven explosions : Insights into Strombolian volcanic eruptions. J Geophys Res 117:B02206

Dingwell DB (1996) Volcanic dilemma : flow or blow ? Science 273:1054-1055

Divoux T, Vidal V, Melo F, Géminard J-C (2008) Acoustic emission associated with the bursting of a gas bubble at the free surface of a non-Newtonian fluid. Phys Rev E 77:056310

Fall A, Bertrand F, Ovarlez G, Bonn D (2009) Yield stress and shear banding in granular suspensions. Phys Rev Lett 103:178301

Fee D, Matoza RS (2013) An overview of volcano infrasound: from hawaiian to plinian, local to global. J Volcanol Geoth Res 240:123-139

Gonnermann HM, Manga M (2013) Dynamics of magma ascent in the volcanic conduit. In: Fagents SA, Gregg TKP, Lopes MC (eds) Modeling Volcanic Processes. Cambridge University Press, Cambridge, pp 55-84

Guazzelli E, Pouliquen O (2018) Rheology of dense granular suspensions. J Fluid Mech 852:P1. https://doi.org/10.1017/jfm.2018.548
Gurioli L, Coló L, Bollasina AJ, Harris AJL, Whittington A, Ripepe M (2014) Dynamics of strombolian explosions : inferences from field and laboratory studies of erupted bombs from stromboli volcano. J Geophys Res 119:319-345

Ishibashi H (2009) Non-Newtonian behavior of plagioclase-bearing basaltic magma: subliquidus viscosity measurement of 1707 basalt of Fuji volcano, Japan. J Volcanol Geoth Res 181:78-88

Ishii K, Yokoo A, Kagiyama T, Ohkura T, Yoshikawa S, Inoue H (2019) Gas flow dynamics in the conduit of Strombolian explosions inferred from seismoacoustic observations at Aso volcano, Japan. Earth Planets Space 71:13. https://doi.org/10.1186/s40623-019-0992-z

James MR, Lane SJ, Chouet B, Gilbert J (2004) Pressure changes associated with ascent and bursting of gas slugs in liquid-filled vertical and inclined conduits. J Volcanol Geoth Res 129:61-82

James MR, Lane SJ, Wilson L, Corder SB (2009) Degassing at low magma-viscosity volcanoes: Quantifying the transition between passive bubble-burst and Strombolian eruption. J Volcanol Geoth Res 180:81-88

Johnson JB, Ripepe M (2011) Volcano infrasound: a review. J Volcanol Geoth Res 206:61-69

Johnson JB, Ruiz MC, Oritz HD, Watson LM, Viracucha G, Ramon P, Amleida M (2018a) Infrasound Tornillos produced by Volcan Cotopaxi's deep crater. Geophys Res Lett. https://doi.org/10.1029/2018GL077766

Johnson JB, Watson LM, Palma JL, Dunham EM, Anderson JF (2018b) Forecasting the Eruption of an Open-Vent Volcano using Resonant Infrasound Tones. Geophys Res Lett 45:2213-2220. https://doi.org/10.1029/2017GL076506

Kinsler L, Frey AR, Coppens AB, Sanders JV (2000) Fundamentals of acoustics. John Wiley \& Sons Inc., New York

Kobayashi T, Namiki A, Sumita I (2010) Excitation of airwaves caused by bubble bursting in a cylindrical conduit : Experiments and a model. J Geophys Res 115:B10201

Kulkarny VA (1978) Impulsive discharge noise: balloon burst problem and engine exhaust pulses. J Acoust Soc Am 64:298-301. https://doi.org/10.1121/1. 381975

Larson RG (1999) The structure and rheology of complex fluids. Oxford University Press, New York

Leighton TG (1994) The acoustic bubble. Academic Press, London

Lyons J, Ichihara M, Kurokawa A, Lees JM (2013) Switching between seismic and seismo-acoustic harmonic tremor simulated in the laboratory : Insights into the role of open degassing channels and magma viscosity. J Geophys Res 118:227-289

Lyons JJ, Haney MM, Fee D, Welch AG, Waythomas CF (2019) Infrasound from giant bubbles during explosive submarine eruptions. Nature Geosci 12:952-958

Mader HM, Llewellin EW, Mueller SP (2013) The rheology of two-phase magma: a review and analysis. J Volcanol Geoth Res 257:135-158

Moitra P, Gonnermann HM, Houghton BF, Tiwary CS (2018) Fragmentation and Plinian eruption of crystallizing basaltic magma. Earth Planet Sci Lett 500:97-104

Montalto P, Cannata A, Privetera E, Gresta S, Nunnari G, Patané D (2010) Towards an automatic monitoring system of infrasonic events at Mt. Etna: strategies for source location and modeling. Pure Appl Geophys 167:1215-1231

Murase T, McBirney AB (1973) Properties of some common igneous rocks and their melts at high temperatures. Geol Soc Am Bull 84:3563-3592

Namiki A, Tanaka Y (2017) Oscillatory rheology measurements of particle- and bubble-bearing fluids: solid-like behavior of a crystal-rich basaltic magma. Geophys Res Lett 44:8804-8813

Namiki A, Tanaka Y, Yokoyama T (2018) Physical characteristics of scoriae and ash from 2014-2015 eruption of Aso Volcano Japan. Earth Planets Space 70:147. https://doi.org/10.1186/s40623-018-0914-5

Nguyen CT, Gonnermann HM, Chen Y, Huber C, Maiorano AA, Gouldstone A, Dufek J (2013) Film drainage and the lifetime of bubbles. Geochem Geophys Geosys 14:3616-3631

Philpotts AR, Ague JJ (2009) Principles of igneous and metamorphic petrology. Cambridge University Press, Cambridge

Podolskiy EA (2020) Toward the acoustic detection of two-phase flow patterns and Helmholtz resonators in englacial drainage systems. Geophys Res Lett 47:e2020GL086951

Poujol M, Wunenburger R, Ollivier F, Antkowiak A, Pierre J (2021) Sound of effervescence. Phys Rev Fluids 6:013604 
Spiel DE (1992) Acoustical measurements of air bubbles bursting at a water surface: bursting bubbles as Helmholtz resonators. J Geophys Res 97(C7):11443-11452

Sumita I, Manga M (2008) Suspension rheology under oscillatory shear and its geophysical implications. Earth Planet Sci Lett 269:467-476

Taylor G (1959) The dynamics of thin sheets of fluid. III, Disintegration of fluid sheets. Proc R Soc London Ser A 253:313-321. https://doi.org/10.1098/rspa. 1959.0196

Tran A, Rudolph ML, Manga M (2015) Bubble mobility in mud and magmatic volcanoes. J Volcanol Geoth Res 294:11-24

Tsunematsu K, Ishii K, Yokoo A (2019) Transport of ballistic projectiles during the 2015 Aso Strombolian eruption. Earth Planets Space 71:49. https://doi.org/ 10.1186/s40623-019-1029-3

Vergniolle S, Brandeis G (1994) Origin of the sound generated by Strombolian explosions. Geophys Res Lett 21:1959-1962

Vergniolle S, Caplan-Aucherbach J (2004) Acoustic measurements of the 1999 basaltic eruption of Shishaldin volcano, Alaska 2. Precursor to the Subplinian phase. J Volcanol Geoth Res 137:135-151

Vidal V, Géminard J-C, Divoux T, Melo F (2006) Acoustic signal associated with the bursting of a soap film which initially encloses an overpressurized cav ity. Eur Phys J B 54:321-339. https://doi.org/10.1140/epjb/e2006-00450-0
Vidal V, Ichihara M, Ripepe M, Kurita K (2009) Acoustic waveform of continuous bubbling in a non-Newtonian fluid. Phys Rev E 80:066314

Vidal V, Ripepe M, Divoux T, Legrand D, Géminard J-C, Melo F (2010) Dynamics of soap bubble bursting and its implication to volcano acoustics. Geophys Res Lett 37:L07302. https://doi.org/10.1029/2009GL042360

Wadsworth FB, Witcher T, Vasseur J, Dingwell DB, Scheu B (2017) When does magma break? Adv Volcanol. https://doi.org/10.1007/11157_2017_23

Wiederseiner S, Andreini N, Epely-Chauvin G, Ancey C (2011) Refractive-index and density matching in concentrated particle suspension: a review. Exp Fluids 50:1183-1206

Wilson L (1980) Relationships between pressure, volatile content and ejecta velocity in three types of volcanic explosion. J Volcanol Geoth Res 8:297-313

Yokoo A, Ishii K, Ohkura T, Kim K (2019) Monotonic infrasound waves observed during the 2014-2015 eruption of Aso volcano, Japan. Earth Planets Space 71:12. https://doi.org/10.1186/s40623-019-0993-y

\section{Publisher's Note}

Springer Nature remains neutral with regard to jurisdictional claims in published maps and institutional affiliations.

\section{Submit your manuscript to a SpringerOpen ${ }^{\odot}$ journal and benefit from:}

- Convenient online submission

- Rigorous peer review

- Open access: articles freely available online

- High visibility within the field

- Retaining the copyright to your article

Submit your next manuscript at $\boldsymbol{\nabla}$ springeropen.com 